\title{
Chemical and Enzymatic Methods for Preparing Circular Single-Stranded DNAs
}

Each of the following three protocols for preparing single-stranded DNA (ssDNA) circles from 28 to at least 188 nucleotides (nt) in length involves a "one-pot" cyclization scheme using various chemical ligation reagents or enzymes and short synthetic DNA splints. Each ligation can proceed from two or more short linear DNAs (precursor segments), which are intermolecularly ligated to give a linear precircle. This DNA is then intramolecularly ligated to form a circular DNA. Alternatively, a single full-length cyclization starting material can be used, again using intramolecular ligation conditions to close it. Enzymatic ligation (see Basic Protocol) is based on the use of a double-helical splint complex with a complementary ssDNA splint (Fig. 5.2.1). This complex assists enzymatic ligation by juxtaposing reactive ends of precursor segments for subsequent ligation to a ssDNA circle using T4 DNA ligase. Chemical ligation with cyanogen bromide (see Alternate Protocol 1) involves an additional, alternative method of assistance through a triple-helical splint complex (Fig. 5.2.1). Chemical ligation occurs by simultaneous dimeric ligation of linear DNAs to circular form upon addition of cyanogen bromide. The final procedure (see Alternate Protocol 2) is a novel chemical autoligation method that also uses a double-helical splint complex. The reaction proceeds via nucleophilic displacement of iodide from 5'-iodothymidine by the 3 '-phosphorothioate group to create a $5^{\prime}$-bridging phosphorothioate linkage. A series of five support protocols cover the preparation and purification (optional) of the end-modified DNAs required for all ligation methods.

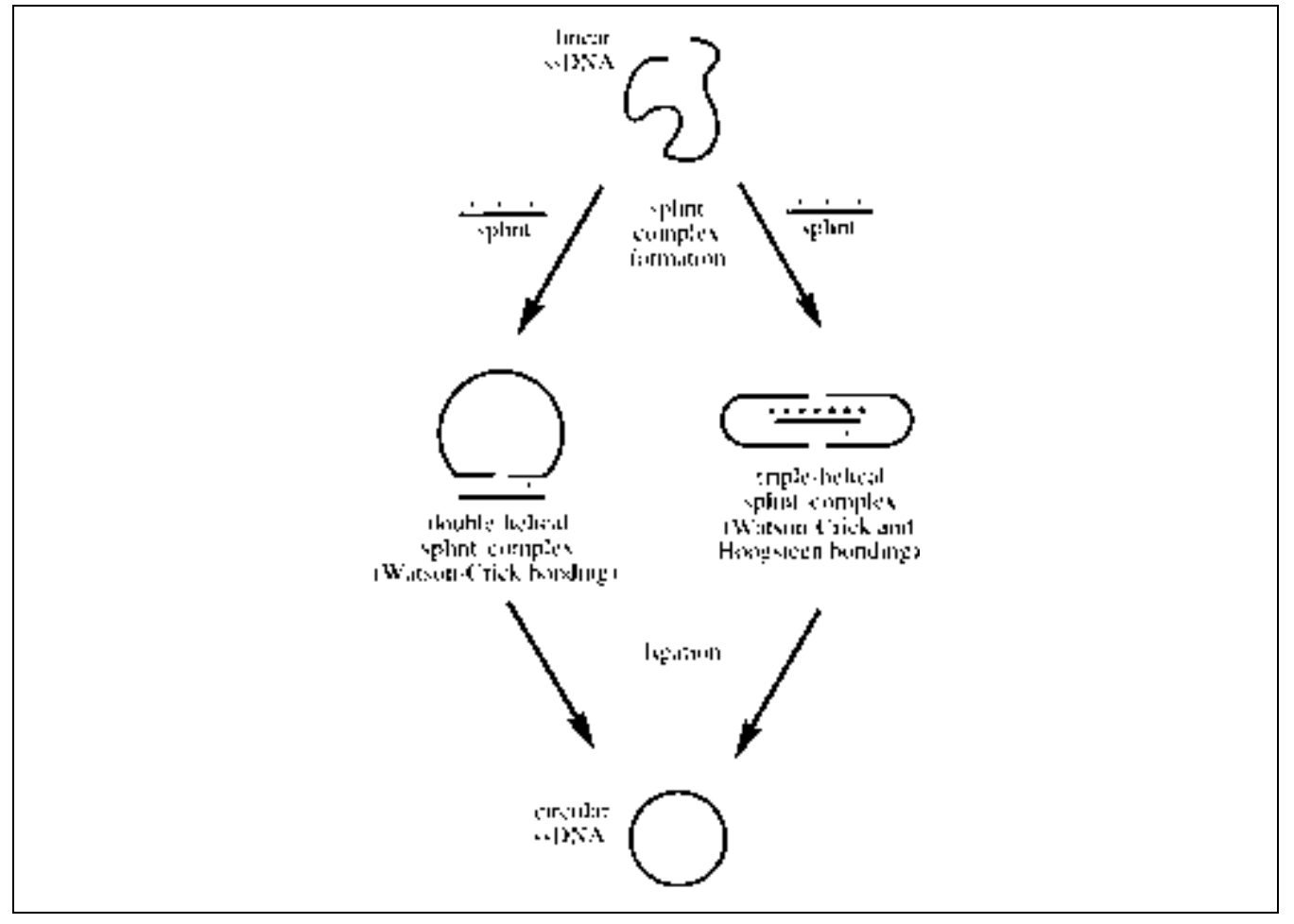

Figure 5.2.1 Cyclization of ssDNAs requires a splint to assist in end joining. Representation of two types of splint complexes used for ligation: double-helical splint complex using conventional Watson-Crick hydrogen bonding, and triple-helical splint complex using both Watson-Crick and Hoogsteen hydrogen bonding. 


\section{STRATEGIC PLANNING}

Each protocol can be used for the synthesis of single-stranded circles ranging in size from 28 to at least $188 \mathrm{nt}$. The enzymatic ligation method has no inherent sequence restrictions; however, the autoligation method requires a $5^{\prime}$-iodothymidine and $3^{\prime}$-phosphorothioate group at the ligation junction, which in turn requires preparation of this DNA using a modified phosphoramidite, 5-iodothymidine, as well as a sulfurizing reagent. The chemical ligation method using cyanogen bromide has been very effective for the synthesis of variously sized circles by dimeric ligation of triple-helical splint complexes (Ruben et al., 1995).

It is possible to prepare circles by any of the three ligation methods; however, one method may be more suitable for a given circle based on certain considerations. Figure 5.2.2 is a decision tree designed to help in choosing a protocol. The first decision involves the size of the circle. Circles $<28 \mathrm{nt}$ are not covered by these protocols, and can best be prepared using methodology described by Alazzouzi et al. (1997). Small circles, 28 to $50 \mathrm{nt}$, are most easily synthesized using a single precursor segment and any of the three basic protocols, with particular steps omitted as noted (Fig. 5.2.2). Medium-sized circles, 50 to $140 \mathrm{nt}$, should be synthesized from two smaller precursor segments, as preparative yields for convergent or dimeric synthesis of this sort are usually significantly better than those obtained using single precursors. Any of the three protocols can be used for the synthesis of medium-sized circles-be sure to note the specific sequence and size considerations for each protocol, however. Large circles, $>140 \mathrm{nt}$, are best prepared from at least three precursor segments. The basic two-step ligation protocol is the same for the synthesis of these large circles; however, all precursor segments and all but one splint are included in the first ligation and the last splint is incorporated in the second ligation. Along with the

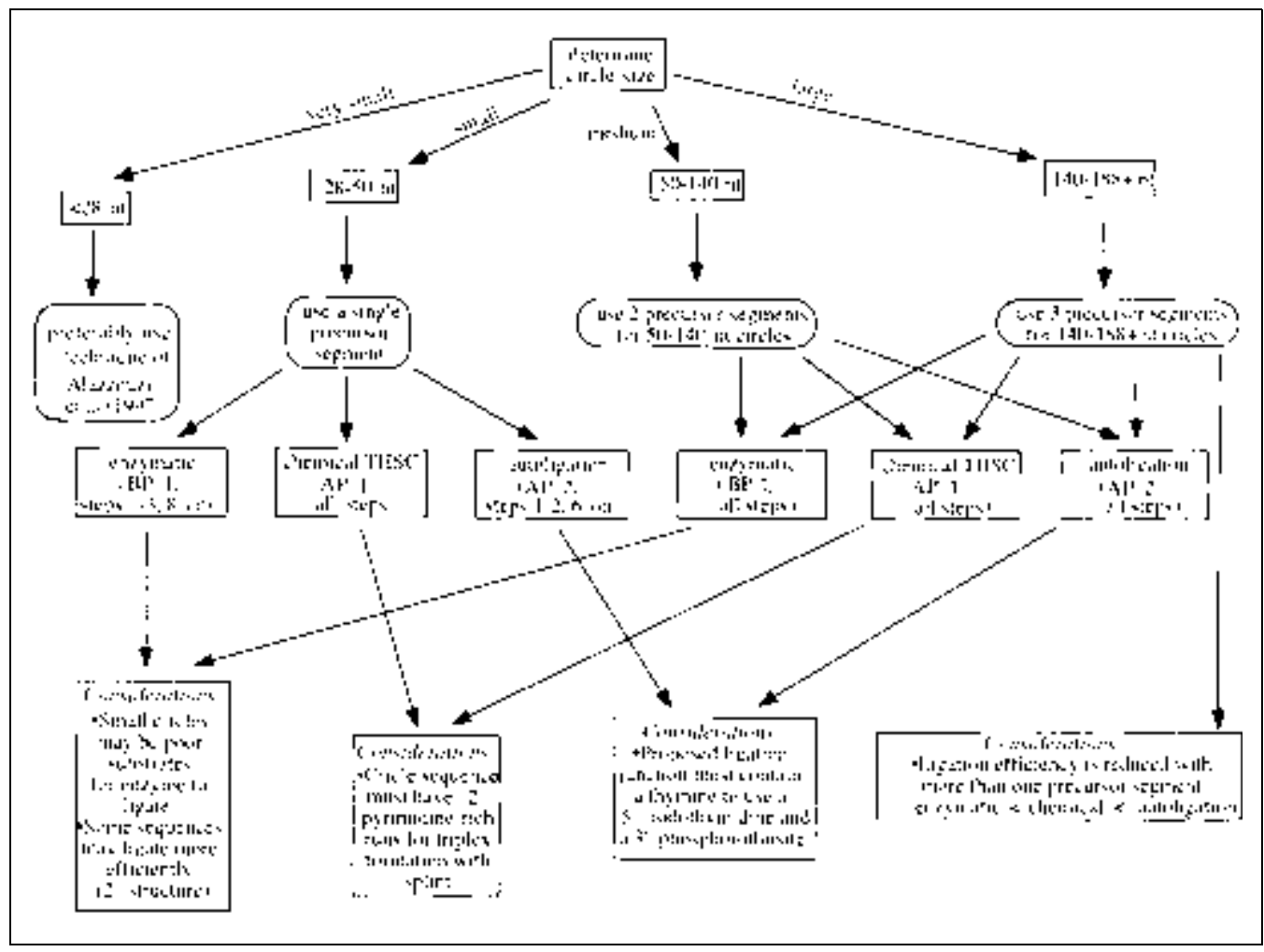

Chemical and Enzymatic Methods
Figure 5.2.2 Strategic planning for DNA circle synthesis using a decision tree. First decision is circle size. This decision will determine the number of precursor segments to use. The next decision involves analysis of sequence requirements and choice of a ligation method. AP, Alternate Protocol; BP, Basic Protocol; THSC, triple-helical splint complex. 


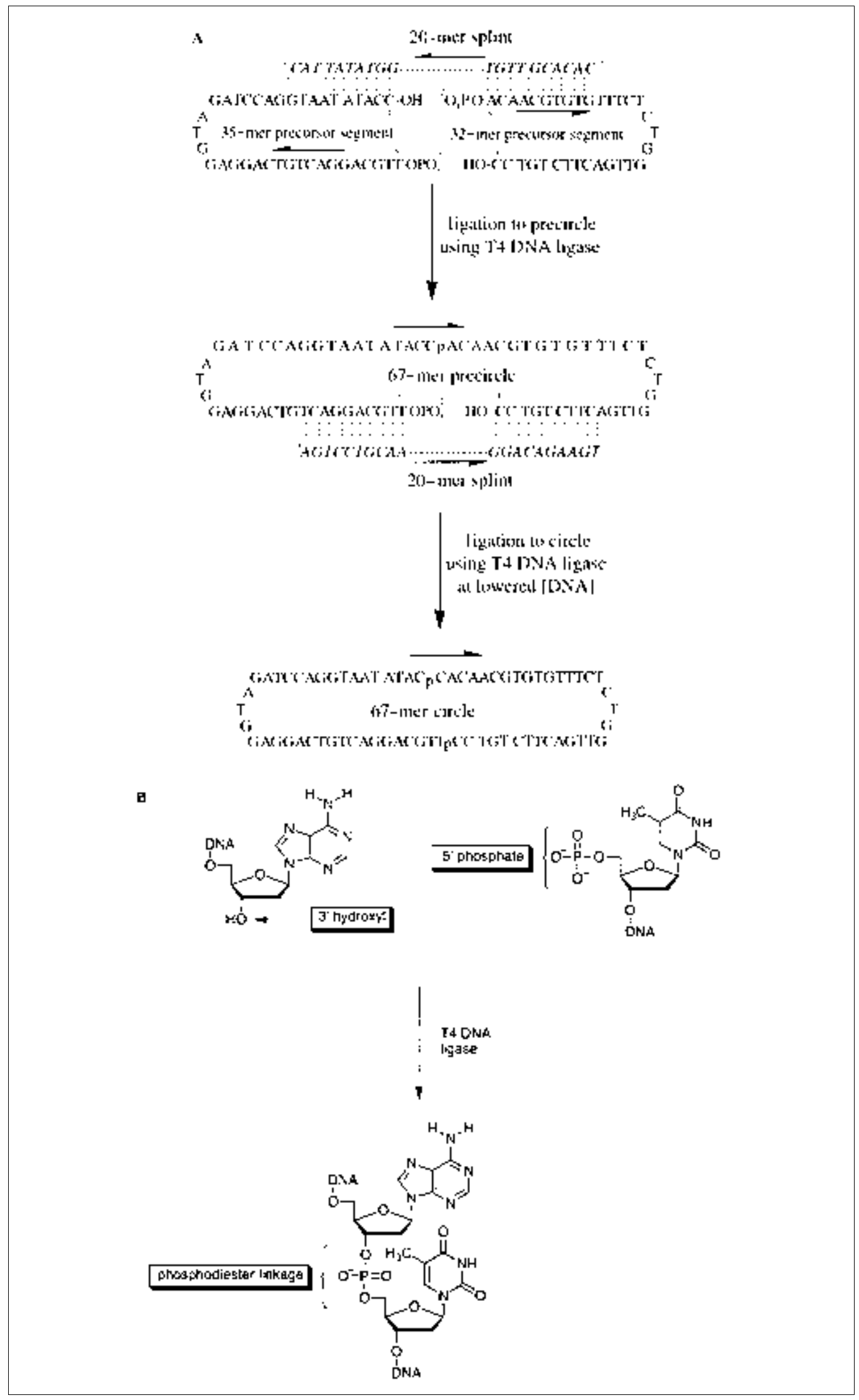

Figure 5.2.3 Schematic representation of the steps in "one-pot" ligation for the enzymatic cyclization of ssDNAs by T4 DNA ligase. (A) Complementary splints 20-nt long are used to form double-helical splint complexes to juxtapose reactive 3'-hydroxyl and 5'-phosphate ends of linear ssDNAs for enzymatic ligation to a phosphodiester linkage (B) using T4 DNA ligase.

Methods for Cross-Linking Nucleic Acids 
specific considerations for each of the three protocols, it must be kept in mind that synthesis of large circles from multiple precursor segments proceeds with low efficiency; the enzymatic method gives the lowest yields, behind the chemical and autoligation methods, whose yields are somewhat better and fairly comparable to one another.

Both the enzymatic and reagent-free ligation protocols operate on a 10- to 15-nmol scale and can easily be scaled up to 100 to $150 \mathrm{nmol}$. However, scales $>150 \mathrm{nmol}$ involve rather large volumes for lyophilization and can become quite costly.

NOTE: If the cyclized oligonucleotides are to be transcribed, all solutions and equipment coming into contact with DNA must be autoclaved to be free of RNase contaminants. All water used should be ultrapure (at least distilled and deionized).

BASIC PROTOCOL
Chemical and Enzymatic Methods

\section{DOUBLE-HELICAL SPLINT COMPLEX-ASSISTED ENZYMATIC CYCLIZATION OF OLIGONUCLEOTIDES USING T4 DNA LIGASE}

The basic protocol describes synthesis and purification of ssDNA circles using a complementary short ssDNA template (a splint) to juxtapose the reactive $3^{\prime}$-hydroxyl and $5^{\prime}$-phosphate groups of precursor segments for ligation by T4 DNA ligase. The protocol is applicable to circles in the size range of 28 to at least 188 nucleotides. This "one-pot" synthesis consists of a two-step ligation of two approximately equal-length precursor segments, which are first enzymatically ligated into a full-length precircle, without isolation, and subsequently enzymatically cyclized into a ssDNA circle (Fig 5.2.3). Circles $<50 \mathrm{nt}$ may be synthesized from a single precursor segment by a single enzymatic ligation and circles $>140 \mathrm{nt}$ by ligation of more than two precursor segments; see Strategic Planning for more information. The products are separated by preparative denaturing polyacrylamide gel electrophoresis (PAGE), and circularity is confirmed by endonuclease cleavage.

CAUTION: Acrylamide and $N, N^{\prime}$-methylenebisacrylamide are neurotoxins. Preparation of solutions with these compounds should be performed in a well-ventilated fume hood, and extreme precautions taken to minimize contact with solids or solutions.

NOTE: To synthesize larger quantities, the first ligation reaction can be scaled up. The second ligation reaction can also be scaled up, but multiple reaction tubes should be used rather than a larger-scale one-pot synthesis.

NOTE: For cyclization using a single precursor segment, skip steps 4 to 7 (i.e., omit the first ligation and proceed directly from DNA quantitation to the second ligation described).

\section{Materials}

20 nt splint ssDNA(s)

Precursor segments containing $5^{\prime}$-phosphate and $3^{\prime}$-hydroxyl groups

Ultrapure (e.g., distilled, deionized) water

$2 \times$ ligation buffer: $20 \mathrm{mM} \mathrm{MgCl} / 100 \mathrm{mM}$ Tris. $\mathrm{Cl}(\mathrm{pH} 7.5)$

$1 \mathrm{M}$ dithiothreitol (DTT; APPENDIX 2A)

$25 \mathrm{mM}$ and $100 \mathrm{mM}$ adenosine triphosphate (ATP)

$400 \mathrm{U} / \mu \mathrm{L}$ T4 DNA ligase (New England Biolabs)

$1 \times$ TBE (APPENDIX 2A)

$10 \%$ denaturing polyacrylamide gel mix (see recipe or purchase)

$10 \%(\mathrm{w} / \mathrm{v})$ ammonium persulfate (APS)

TEMED or TMEDA (Life Technologies)

Formamide loading buffer (see recipe) 
$0.2 \mathrm{~N} \mathrm{NaCl}$

$5 \times \mathrm{S} 1$ nuclease buffer: $50 \mathrm{mM} \mathrm{NaCl} / 50 \mathrm{mM} \mathrm{NaOAc} / 5 \mathrm{mM} \mathrm{ZnCl}, \mathrm{pH} 4.6$

$332 \mathrm{U} / \mu \mathrm{L}$ S1 nuclease (from Aspergillus oryzae; Amersham Pharmacia Biotech)

Stop solution (see recipe)

Stains-all dye solution (see recipe)

UV spectrophotometer

1.5-mL microcentrifuge tubes and cap locks, autoclaved

15-mL screw-top centrifuge tubes, autoclaved

$90^{\circ} \mathrm{C}$ heat block or thermal cycler

Glass wool

Dialysis tubing, MWCO 1000 (e.g., SpectraPor)

Gel electrophoresis equipment:

Vertical (sequencing) gel stand

2000-V power supply

Glass plates: $13 \times 15.5 \mathrm{~cm}, 13 \times 16.5 \mathrm{~cm}, 6.5 \times 15 \mathrm{~cm}$, and $6.5 \times 16 \mathrm{~cm}$

Gel combs: $1.5-\mathrm{mm}$-thick with $1.5-\mathrm{cm}$-wide wells and $0.4 \mathrm{~mm}$ thick with

5-mm-wide wells

Spacers: $1.5 \mathrm{~mm}$ thick and $0.4 \mathrm{~mm}$ thick

UV shadow box or light source

Lyophilizer or SpeedVac

Saran Wrap (or other UV-transparent plastic wrap)

Razor blades, sterile

Glass stir rod

50-mL filter tubes with $0.45-\mu \mathrm{m}$ cellulose acetate filter (e.g., Spin-X II, Corning Costar)

\section{Obtain splint DNAs}

1. Construct or purchase ssDNAs of the desired splint and precursor-segment sequences. Splint sequences should bridge, by $10 \mathrm{nt}$ on each side, the gap between precursor segments for ligation (Fig. 5.2.3A).

For ssDNAs purchased commercially, splints should be fully deprotected and precursor segments should be fully deprotected and 5'-phosphorylated. Alternatively, ssDNAs may be constructed on a DNA synthesizer using the standard DNA cycle and a 0.2- $\mu$ mol scale; see Support Protocol 1 for synthesis of modified precursor segments, see Support Protocol 4 for deprotection, and see Support Protocol 5 for (optional) purification.

\section{Quantitate DNA}

2. Redissolve each DNA in $1.00 \mathrm{~mL}$ water. Prepare a $100 \times$ dilution sample by combining $10.00 \mu \mathrm{L}$ of this stock solution with $990 \mu \mathrm{L}$ water.

Synthesized DNA after deprotection and lyophilization should be powdery and white and should readily redissolve in water. If the solution does not appear homogeneous, mild heating $\left(\sim 60^{\circ} \mathrm{C}\right)$ and/or brief sonication $(1 \mathrm{~min})$ can be used to help dissolve the DNA. Insoluble material may cause some turbidity but is not cause for concern.

3. Use a properly calibrated UV spectrophotometer to obtain a measurement of the absorbance at $260 \mathrm{~nm}\left(A_{260}\right)$ of the $100 \times$ dilution sample prepared in step 2. Calculate the concentration $(c)$ of DNA for the stock sample prepared in step 2 in moles per milliliter (recalling the dilution factor from step 2 of $10^{2}$ ) using Beer's Law:

$$
\begin{aligned}
& A_{260}=\varepsilon \times b \times c \\
& b \text { is generally } 1 \text {, so } c(\text { in } \mathrm{mol} / \mathrm{mL})=\left(A_{260} \times 10^{2}\right) / \varepsilon
\end{aligned}
$$

Methods for Cross-Linking Nucleic Acids 
Extinction coefficients ( $(\varepsilon)$ for each sequence can be calculated using the nearest-neighbor method (Borer, 1985). See also UNIT 7.3.

IMPORTANT NOTE: If a single precursor segment is to be used (i.e., synthesis of a ssDNA circle $<50 \mathrm{nt}$ ), omit the first ligation (steps 4 to 7) and begin with step 8.

\section{Perform first ligation}

4. Combine $125.0 \mu \mathrm{L}$ of $2 \times$ ligation buffer with $15 \mathrm{nmol}$ of each precursor segment and $18.3 \mathrm{nmol}$ of one of the splints (splint 1 ) in an autoclaved $1.5-\mathrm{mL}$ microcentrifuge tube.

Final concentrations: $10 \mathrm{mM} \mathrm{MgCl}$, $50 \mathrm{mM}$ Tris $\cdot \mathrm{Cl}$, $60 \mu \mathrm{M}$ each precursor segment, 73 $\mu M$ splint 1 .

IMPORTANT NOTE: For large DNA circles (>140 nt), use all precursor segments and all but one splint in step 4.

It may not matter which splint is chosen for the first ligation here; however, more is required for the second ligation, so if one is limiting, it should be used here. If the combined volume of DNA (two precursor segments and splint) exceeds $119 \mu \mathrm{L}$, one or more DNAs must be lyophilized to reduce the volume.

5. Calculate the volume of water to add according to the following equation:

volume water $=250 \mu \mathrm{L}-(125.0 \mu \mathrm{L}+$ volume DNA [step 4$]+5.40 \mu \mathrm{L})$

Add the calculated amount of water to the microcentrifuge tube, vortex briefly, and secure closed with a cap lock.

The $250 \mu \mathrm{L}$ represents the total reaction volume, $125.0 \mu \mathrm{L}$ represents the $2 \times$ ligation buffer, volume DNA represents the combined volumes of precursor segments and splint, and 5.40 $\mu L$ represents volumes of reagents to be added in step 7.

6. Incubate the capped tube $10 \mathrm{~min}$ in a $90^{\circ} \mathrm{C}$ heat block. After $10 \mathrm{~min}$, turn the heat block off and allow it to slowly cool, with its contents, to room temperature $\left(\sim 25^{\circ} \mathrm{C}\right)$.

Step 6 takes $\sim 2$ to $2.5 \mathrm{hr}$ to complete. Alternatively, a PCR thermal cycler can be used. Set the cycler to heat for $10 \mathrm{~min}$ at $90^{\circ} \mathrm{C}$, then slowly cool $\left(0.5^{\circ} \mathrm{C} / \mathrm{min}\right)$ to room temperature. The slow cooling allows the splints to bind their complementary targets efficiently, aligning reactive ends for ligation by the enzyme.

7. When the block and its contents have reached room temperature $\left(\sim 25^{\circ} \mathrm{C}\right)$, add 2.50 $\mu \mathrm{L}$ of $1 \mathrm{M}$ DTT, $1.00 \mu \mathrm{L}$ of $25 \mathrm{mM}$ ATP, and $1.88 \mu \mathrm{L}$ of $400 \mathrm{U} / \mu \mathrm{L}$ T4 DNA ligase to the tube. Gently invert the tube and let stand 4 to $6 \mathrm{hr}$ at room temperature.

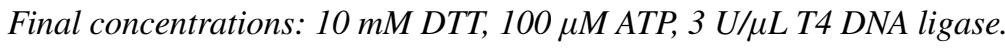

\section{Perform second ligation}

8. In autoclaved $15-\mathrm{mL}$ centrifuge tube, combine $5.0 \mathrm{~mL}$ of $2 \times$ ligation buffer with either $167.0 \mu \mathrm{L}$ of reaction from step $7(2 / 3 \mathrm{vol})$ or $10 \mathrm{nmol}$ full-length synthesized precursor segment (if cyclization is from a single precursor segment), plus $30 \mathrm{nmol}$ of the remaining splint (splint 2).

Final concentrations: $10 \mathrm{mM} \mathrm{MgCl}, 50 \mathrm{mM}$ Tris $\cdot \mathrm{Cl}, 1 \mu \mathrm{M}$ precircle, $3 \mu \mathrm{M}$ splint 2, $1.2 \mu \mathrm{M}$ splint 1 .

IMPORTANT NOTE: For circles $>140$ nt, the final splint is added in this step.

The $167.0 \mu \mathrm{L}$ of reaction from step 7 represents, in theory, a maximum yield of $10 \mathrm{nmol}$ precircle from ligated precursor segments. Alternatively, if the circle size is $<50 \mathrm{nt}, 10 \mathrm{nmol}$ of full-length synthesized precursor segment can be used. Note that DNA concentrations are much less for this second ligation (1 $\mu \mathrm{M}$ precircle) than for the first ligation (60 $\mu \mathrm{M}$ 
each precursor segment). This favors the intramolecular cyclization reaction over an intermolecular reaction that produces undesired multimeric linear species.

9. Calculate the volume of water to add according to the following equation:

volume water $=10,000 \mu \mathrm{L}-(5000 \mu \mathrm{L}+$ volume DNA [step 8] $+118.3 \mu \mathrm{L})$

Add this volume of water to the $15-\mathrm{mL}$ tube(s), vortex briefly, and secure closed.

The 10,000 $\mu \mathrm{L}$ represents the total reaction volume; $5000 \mu \mathrm{L}$ represents the $2 \times$ ligation buffer; volume DNA represents the combined volumes of either $167.0 \mu \mathrm{L}$ of step 7 reaction or the synthesized precursor segment, plus splint; and $118.3 \mu \mathrm{L}$ represents volumes of reagents to be added later. Normally it will not be necessary to lyophilize any of the components at this step, as the calculated volume of water often exceeds $4.5 \mathrm{~mL}$.

10. Wrap the tube(s) thoroughly with glass wool and incubate $10 \mathrm{~min}$ in a $90^{\circ} \mathrm{C}$ heat block, inverting them every $2 \mathrm{~min}$. After $10 \mathrm{~min}$, turn the heat block off and allow it to cool, with its contents, to room temperature $\left(\sim 25^{\circ} \mathrm{C}\right)$.

The glass wool will allow even heating and cooling of the reagents to ensure an efficient slow annealing of splint and precircle. Inverting the tubes also ensures thorough heating of the contents. As before, this slow annealing takes $\sim 2$ to $2.5 \mathrm{hr}$ to complete.

11. When the block and its contents have reached room temperature $\left(\sim 25^{\circ} \mathrm{C}\right)$, add 100.0 $\mu \mathrm{L} 1 \mathrm{M}$ DTT, $10.00 \mu \mathrm{L}$ of $100 \mathrm{mM}$ ATP, and $8.33 \mu \mathrm{L}$ of $400 \mathrm{U} / \mu \mathrm{L}$ T4 DNA ligase to each tube. Gently invert the tube and allow reaction to proceed 12 to $16 \mathrm{hr}$ at room temperature.

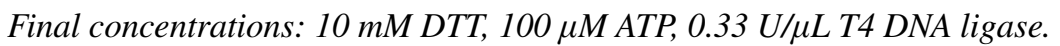

Note the different concentrations of enzyme and DNA used here compared to the first ligation. While the final ATP and DTT concentrations remain the same, the enzyme and DNA concentrations are lowered to favor the intramolecular cyclization reaction and to conserve enzyme and thus decrease cost.

\section{Dialyze reaction products}

12. Transfer the entire reaction volume from step 11 to MWCO 1000 dialysis tubing clamped at one end to make a bag. Secure the other end of the dialysis bag closed. Dialyze against $2 \mathrm{~L}$ ultrapure water for $3 \mathrm{hr}$. Repeat.

13. Lyophilize the dialysate to dryness.

The dried residue should look off-white in color.

\section{Gel purify reaction products}

14. Prepare a vertical, 1.5 -mm-thick (preparative), denaturing $10 \%$ polyacrylamide gel using a comb that produces 1.5 -cm-wide wells.

A gel prepared between plates used for sequencing $(13 \times 16.5$ inch $)$ allows for ample vertical loading of reaction mixture and sufficient migration length for separation and resolution of bands. For these conditions, prepare gel solution from $250 \mathrm{~mL}$ of $10 \% \mathrm{gel}$ mix, $1.67 \mathrm{~mL}$ of 10\% APS, and $53.3 \mu \mathrm{L}$ TEMED. The gel should contain $\sim 12$ wells for reaction solution plus a few for DNA markers. For more detailed description of procedures for denaturing PAGE, see APPENDIX $3 B$.

15. Place the gel in a gel electrophoresis apparatus, using $1 \times$ TBE in both top and bottom buffer reservoirs, and prerun $30 \mathrm{~min}$ at $30 \mathrm{~W}$ constant power, room temperature.

16. Redissolve each $10 \mathrm{~mL}$ lyophilized sample (from second ligation, step 13) in $60 \mu \mathrm{L}$ water and $60 \mu \mathrm{L}$ formamide loading buffer by brief vortexing. 
If the solution does not appear homogeneous, mild heating $\left(\sim 60^{\circ} \mathrm{C}\right)$ and/or brief sonication (1 min) can be used to help dissolve the DNA mixture.

17. Load $10 \mu \mathrm{L}$ of the solution from step 16 in each of the $1.5-\mathrm{cm}$-wide wells. Load DNA markers, step 2 solutions, and first ligation-reaction mixture (step 7) in separate adjacent wells (use $\sim 1 \mathrm{nmol}$ of each to permit later visualization by UV shadowing).

Use of DNA markers allows for confirmation of product lengths. Precursor segments (step 2) and solution from step 7 (first ligation) can be used to identify precursor segments and precircles in the reaction lanes.

18. Run the gel at $30 \mathrm{~W}$ constant power, room temperature, to the desired length.

On a 10\% gel bromphenol blue migrates similarly to a 26-nt linear DNA, so this band is usually run off, since most circles will be larger. Xylene cyanol runs like a 55-nt linear $D N A$, and for larger circles (>70 nt) should be run almost off the gel.

19. Disassemble the gel apparatus. Sandwich the prep gel between two pieces of Saran Wrap. View the gel under ultraviolet light with fluorescent white background (glass silica plates work well) to visualize the bands.

The reaction lanes should show several bands. Figure 5.2.4 is a representation of a typical gel, with lane 6 containing second ligation reaction mixture. Depending on how far the gel is run, the splints (lane 1) will probably be run off, but the precursor segments may be visible and their identity can be confirmed by their co-migration with the precursor-segment markers (step 2 solutions) run alongside (lanes 2 and 3). The next band up (migrating more slowly) from the precursor segments should be the precircle band; this is confirmed by its co-migration with the largest band from the first ligation reaction (step 7) run alongside (lane 4). Although the secondary structure present in many DNA circles may cause them to migrate more slowly than their linear counterparts (Serwer and Allen, 1984), identification of the circle (lane 8) can be difficult, as there may be one or more products in between the linear precircle and monomer circle. These are likely to be ligation of an odd number of precursor segments (lane 7), and results are especially complex if precursor segments are of different sizes. Migration of circles is not always consistent and can be temperature dependent. Therefore, most bands migrating more slowly than confirmed precircle should be cut out for analysis.

\section{Isolate purified DNAs after gel electrophoresis}

20. Cut out the desired band with a clean, sterile razor blade and place the pieces in a 15-mL centrifuge tube. Using a glass stir rod, crush the gel pieces thoroughly. Combine crushed pieces with $\sim 5$ to $10 \mathrm{~mL}$ of $0.2 \mathrm{~N} \mathrm{NaCl}$ to make an easily shaken slurry. Repeat, in separate tubes, for each band of interest isolated. Shake each slurry for $\sim 12 \mathrm{hr}$.

Precircle and higher bands should be isolated for reasons stated in step 19. Be sure all crushed gel pieces are transferred to the slurry. Conversion of precircle to circle may appear high by UV shadowing (step 19), but isolated yields after step 22 are often $<50 \%$; it is therefore important to ensure quantitative transfer of all materials.

21. Filter the slurry by brief (1- to 5-min) centrifugation through $50-\mathrm{mL}$ filter tubes. Resuspend the gel pieces in $5 \mathrm{~mL}$ water, centrifuge again, and combine the filtrates.

It is advisable to save the filtered gel pieces until after quantitation, as additional extractions with $0.2 \mathrm{~N} \mathrm{NaCl}$ may be desired to increase isolated product yield, although only minimal $(<10 \%)$ additional recoveries should be expected.

Chemical and Enzymatic Methods

22. Dialyze the filtrate from step 21 as described in step 12, but using $2 \mathrm{~L}$ ultrapure water for $6 \mathrm{hr}$ and repeating three times.

23. Quantitate as described in step 3 using the dialysate from step 22 with no dilution. 


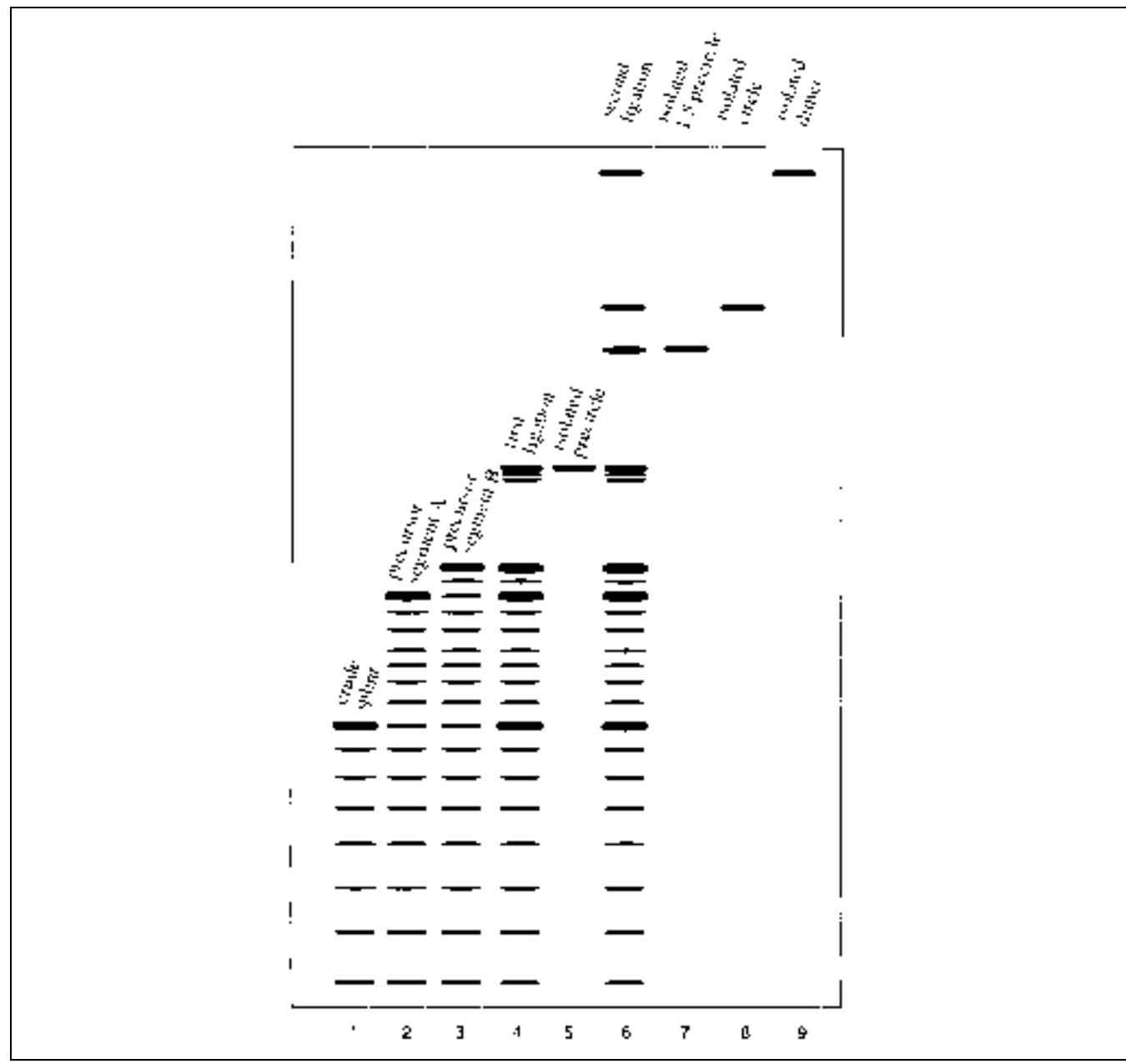

Figure 5.2.4 Representation of a typical cyclization gel. Lanes 1 to 3 represent crude synthesized DNAs used for ligation; banding underneath attests to their impurity. Lane 4 represents the first ligation-reaction mixture; the largest (slowest-migrating) species is the precircle, shown after isolation in lane 5. Lane 6 represents the second ligation (cyclization) and the possible products as confirmed by their migration with isolated species [lanes 7 to $9: 1.5 \times$ precircle (from ligation of 3 precursor segments in a linear fashion), circle and dimer]. Note the migration of the bands isolated in lanes 7 to 9 is typical but may differ slightly with different methods and sequences. Note: While the ligation mechanism selects for correct sequence precursor segments, it is not unusual to see less than full-length impurities ( $n-1, n-2$, etc.), and these impurities are often eliminated by careful isolation of the desired band.

Incorrect identification of bands may result in erroneous concentration calculations from the use of the wrong extinction coefficient. Confirmation of identification will be discussed in the next section. If a misidentification is discovered, simple recalculation with a different extinction coefficient is all that is required to correct the calculation error, as the absorbance is the same regardless of identification.

\section{Confirm circularity of product}

24. Place $\sim 0.2 \mathrm{nmol}$ of the suspected circular DNA in each of two tubes and $\sim 0.2 \mathrm{nmol}$ of isolated precircle in each of two tubes (four tubes total). Label one of each pair "control" and one "S1 nuclease reaction," and lyophilize.

25. To each tube from step 24 , add $0.60 \mu \mathrm{L}$ of $5 \times \mathrm{S} 1$ nuclease buffer. To the control tubes, add $2.50 \mu \mathrm{L}$ water; to the reaction tubes, add $2.00 \mu \mathrm{L}$ water. To the reaction tubes, add $0.50 \mu \mathrm{L}$ diluted $\mathrm{S} 1$ nuclease (prepared from $0.50 \mu \mathrm{L}$ of $332 \mathrm{U} / \mu \mathrm{L}$ enzyme stock +

Methods for Cross-Linking Nucleic Acids 


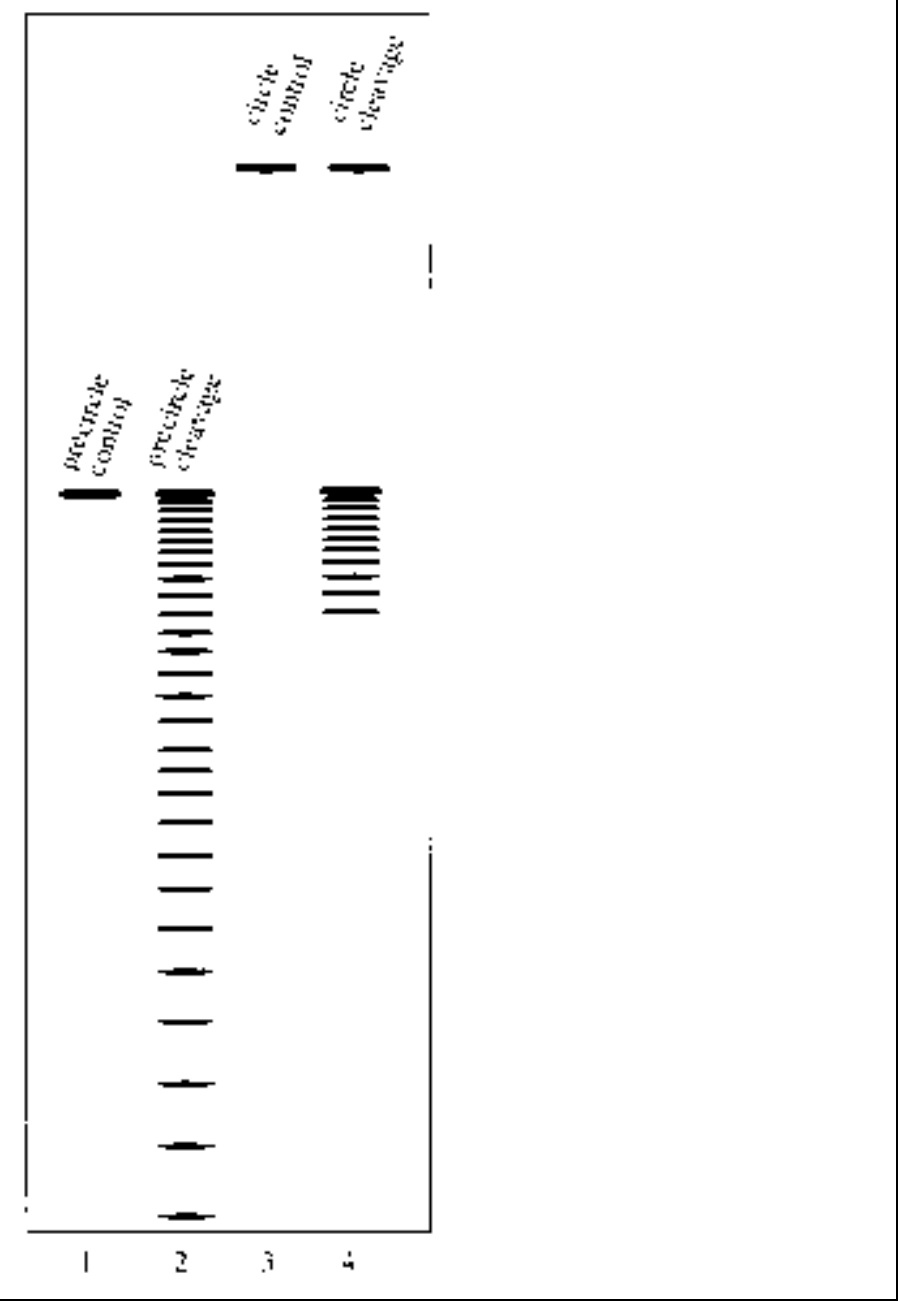

Figure 5.2.5 Representation of a typical characterization gel. Lanes 1 and 3 represent isolated precircle and circle controls. Lanes 2 and 4 represent precircle and circle cleavage by $\mathrm{S} 1$ nuclease, an endonuclease. As would be expected for endonuclease cleavage of a circle (lane 4), the initial cleavage produces a species that exhibits the same migration as the full-length cyclization precursor (precircle). Initial endonuclease cleavage of the linear precircle (see in lane 2 and also lane 4), by contrast, produces a continuous banding pattern.

$373 \mu \mathrm{L}$ water). Incubate all tubes $10 \mathrm{~min}$ at $37^{\circ} \mathrm{C}$. Stop reactions by adding $1 \mathrm{vol}$ $(3.10 \mu \mathrm{L})$ stop solution to each.

26. Prepare a vertical, 0.4 -mm-thick (analytical), denaturing $10 \%$ polyacrylamide gel using a comb that produces $\sim 5$-mm-wide wells. Prerun the gel as in step 15.

Using half-width sequencing plates $(6.5 \times 16$ in) and $5-\mathrm{mm}$ well width comb, the entire reaction volume $(6.20 \mu \mathrm{L})$ can be analyzed. For these conditions, prepare gel solution from $50 \mathrm{~mL}$ of $10 \%$ gel mix, $333 \mu \mathrm{L}$ of $10 \%$ APS, and $10.67 \mu \mathrm{L}$ TEMED.

27. Load the entire reaction volume, $6.20 \mu \mathrm{L}$, on the gel and run dyes to the same distance as for the second ligation purification gel (step 18).

28. When electrophoresis is complete, disassemble the gel apparatus and transfer the analytical gel to a piece of Saran Wrap. Transfer the gel to an ultrapure water bath and soak $10 \mathrm{~min}$ to remove urea.

Chemical and Enzymatic Methods 
29. Remove the water soak and add $200 \mathrm{~mL}$ of Stains-all dye solution. Wait $10 \mathrm{~min}$ for staining of bands.

Background staining can be lightened by exposing the gel to incandescent light $(100 \mathrm{~W}$, held at 1 to 2 feet) while it is immersed in a fresh water bath.

30. Assess circularity of the DNA: this is confirmed by the absence of banding between the circle band and the precircle band (each confirmed by appropriate control).

Initial cleavage of a circle produces a single band with the mobility of the full-length precursor, the precircle, Figure 5.2.5 (compare lanes 1 and 4). If the putative circular DNA is actually linear, as is the case for the precircle reaction lane (lane 2), banding will be seen directly under the suspected band (compare lanes 1 and 2).

\section{TRIPLE-HELICAL SPLINT COMPLEX-ASSISTED CHEMICAL CYCLIZATION OF OLIGONUCLEOTIDES USING CYANOGEN BROMIDE}

The protocol for chemical ligation using cyanogen bromide involves synthesis and purification of ssDNA circles using a pyrimidine-rich triple-helical-forming ssDNA template (a splint) to juxtapose reactive $3^{\prime}$-hydroxyl and 5'-phosphate (or 5'-hydroxyl and $3^{\prime}$-phosphate) ends of linear ssDNAs for chemical ligation using cyanogen bromide. It is noteworthy that unlike in enzymatic ligation (see Basic Protocol), either orientation of phosphate or hydroxyl is usable (i.e., the precursor segments can be either $3^{\prime}$ - or $5^{\prime}$-phosphorylated). The protocol is applicable for triplex-forming circles (TFCs) in the size range of 28 to $\sim 150 \mathrm{nt}$.

The synthesis of TFCs begins with two approximately equal-length precursor segments that are highly pyrimidine rich at the ligation site, allowing for formation of a triple-helical splint complex with a purine-rich splint for simultaneous dimeric ligation into a ssDNA circle (see Fig. 5.2.6A). The basic chemistry of ligation can be either that depicted in Figure 5.2.3B or Figure 5.2.6B. The products are separated by preparative denaturing PAGE, and circularity confirmed by endonuclease cleavage.

CAUTION: Cyanogen bromide is highly toxic and volatile; all work with this compound should be performed in a well-ventilated fume hood.

\section{Additional Materials (also see Basic Protocol)}

One purine-rich, triple-helical-forming splint ssDNA

Two triplex-forming precursor segments containing either $3^{\prime}$ - or $5^{\prime}$-phosphates

$2 \times$ ligation buffer: $200 \mathrm{mM} \mathrm{NiCl} 2 / 400 \mathrm{mM}$ imidazole $\cdot \mathrm{HCl}(\mathrm{pH} 7.0)$

Cyanogen bromide (BrCN; solid)

1. Construct or purchase ssDNAs of the desired splint and precursor-segment sequences. Splint sequence should bridge, by $10 \mathrm{nt}$ on each side, the gap between precursor segments for ligation (see Fig. 5.2.6A).

For ssDNAs purchased commercially, splints should be fully deprotected and precursor segments should be fully deprotected and either 5'-or 3'-phosphorylated. For use of commercially purchased DNAs, proceed to step 2. Alternatively, ssDNAs may be con-

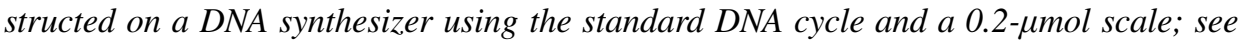
Support Protocol 2 for 3'-phosphate- or Support Protocol 1 for 5'-phosphate-containing precursor segments, Support Protocol 4 for deprotection, and Support Protocol 5 for (optional) purification.

\section{Quantitate DNA}

2. Quantitate DNA (see Basic Protocol, steps 2 and 3).

Methods for Cross-Linking Nucleic Acids

Current Protocols in Nucleic Acid Chemistry 


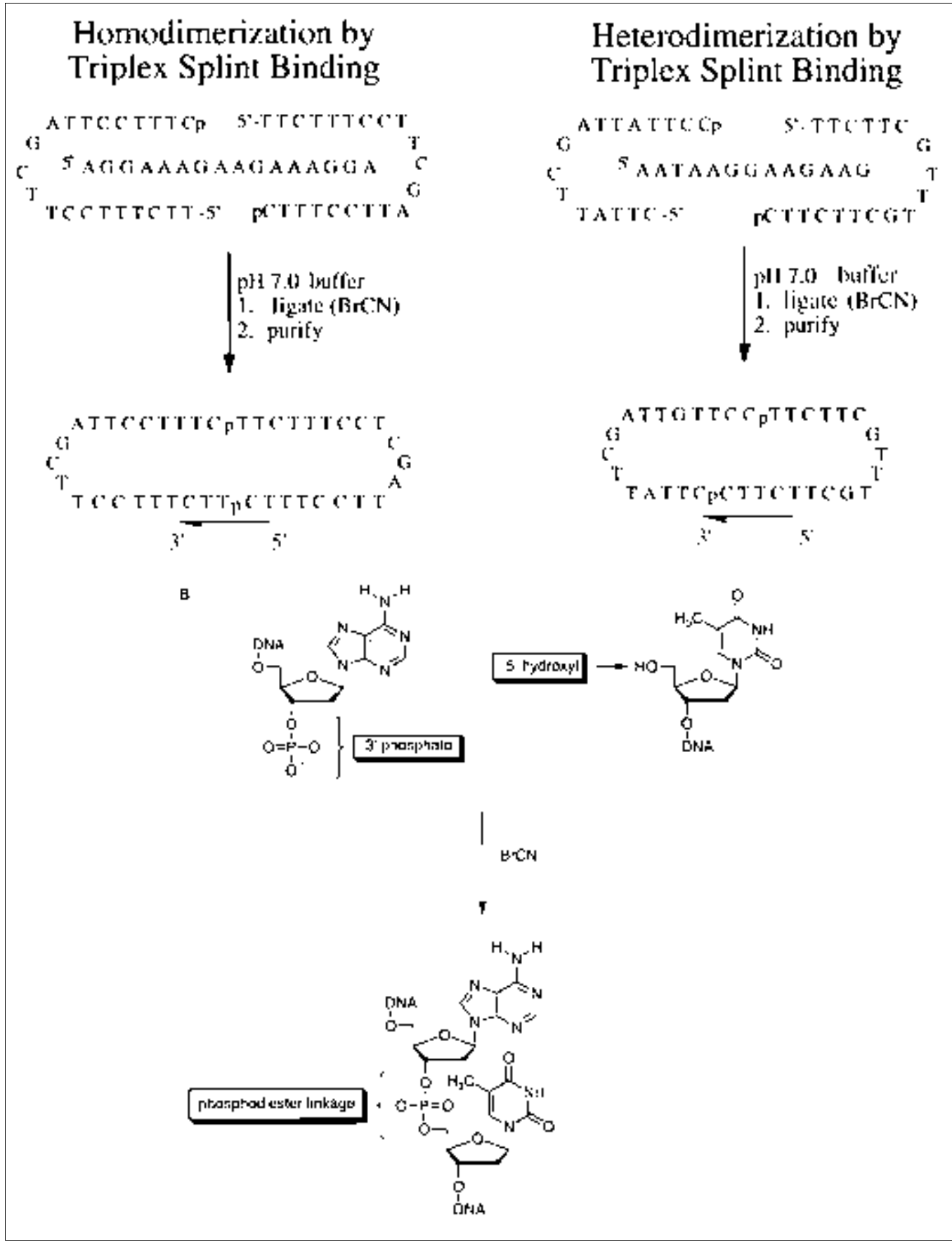

Figure 5.2.6 Schematic representation of ligation steps for the chemical cyclization of ssDNAs using cyanogen bromide, BrCN. (A) A purine-rich ssDNA splint is used to form a triple-helical splint complex to juxtapose reactive $3^{\prime}$-phosphate and 5'-hydroxyl ends of linear DNAs for a simultaneous dimeric ligation to a phosphodiester linkage, (B), using $\mathrm{BrCN}$. Note: For chemical ligation using $\mathrm{BrCN}$, a 5'-phosphate and 3'-hydroxyl such as are used in Figure 5.2.3B are also suitable.

Chemical and Enzymatic Methods

\section{Ligation}

3. Combine $750 \mu \mathrm{L}$ of $2 \times$ ligation buffer with $75 \mathrm{nmol}$ of each precursor segment and $82.5 \mathrm{nmol}$ of the splint in an autoclaved $1.5-\mathrm{mL}$ microcentrifuge tube

Final concentrations: $100 \mathrm{mM} \mathrm{NiCl}, 200 \mathrm{mM}$ imidazole $\mathrm{HCl}, 50 \mu \mathrm{M}$ each precursor segment or precircle, $55 \mu \mathrm{M}$ splint. 
If combined volume of DNA (two precursor segments and splint) exceeds $750 \mu \mathrm{L}$, one or more DNAs must be lyophilized to reduce the volume.

4. Calculate the volume of water to add according to the following equation:

volume water $=1500 \mu \mathrm{L}-(750 \mu \mathrm{L}+$ volume DNA [step 3] $)$

Add the calculated amount of water to the microcentrifuge tube and vortex thoroughly.

The $1500 \mu \mathrm{L}$ represents the total reaction volume, $750 \mu \mathrm{L}$ represents the $2 \times$ ligation buffer, and DNA represents the combined volumes of both precursor segments and the splint.

5. Weigh out $19.9 \mathrm{mg}$ cyanogen bromide ( $125 \mathrm{mM}$ final concentration) and add to the microcentrifuge tube. Thoroughly vortex to completely dissolve cyanogen bromide and allow to react at $23^{\circ} \mathrm{C}$ (room temperature) for $12 \mathrm{hr}$.

CAUTION: Cyanogen bromide is highly toxic; all work with this compound should be performed in a well-ventilated fume hood.

Do not let the reaction proceed beyond $12 \mathrm{hr}$, as degradation of DNA may become problematic.

\section{Dialyze reaction after ligation}

6. Dialyze the ligation-reaction mixture (see Basic Protocol, steps 12 and 13).

\section{Gel purify DNA products}

7. Gel purify the dialyzed mixture (see Basic Protocol, steps 14 to 19).

The reaction lanes should show several bands. Figure 5.2.4 is a representation of a typical gel, with lane 6 containing the ligation-reaction mixture. Depending on how far the gel is run, the splint will probably be run off, but the precursor segments or precircle may be visible and their identity can be confirmed by their co-migration with appropriate DNA markers from step 2 (lanes 1 to 3) run alongside. Although DNA circles usually migrate more slowly than their linear counterparts (Serwer and Allen, 1984), identification of circles is not trivial. Circle migration is not always consistent and can be temperature dependent. Triple-helical splint complexes can yield multimers from ligation of an odd number of precursor segments (lane 7), although the dimeric mechanism for ligation reduces this tendency. However, most bands migrating more slowly than precursor segments or precircle should be cut out for analysis.

\section{Isolate purified DNAs}

8. Isolate purified DNAs from the electrophoretic gel (see Basic Protocol, steps 20 to 23).

Precircle and higher products should be isolated for reasons stated in step 7.

\section{Confirm circularity}

9. Confirm circularity of the desired DNA product (see Basic Protocol, steps 24 to 30).

\section{DOUBLE-HELICAL SPLINT COMPLEX-ASSISTED REAGENT-FREE CYCLIZATION OF OLIGONUCLEOTIDES USING A 3'-PHOSPHOROTHIOATE GROUP AND 5'-IODOTHYMIDINE}

The protocol describes synthesis and purification of ssDNA circles using a complementary ssDNA template (a splint) to create a double-helical splint complex to juxtapose reactive 3 '-phosphorothioate group and $5^{\prime}$-iodothymidine of ssDNAs for autoligation by nucleophilic displacement. The protocol is applicable to circles in the size range of 28 to at least 188 nucleotides. The "one-pot" synthesis consists of two approximately equal- 


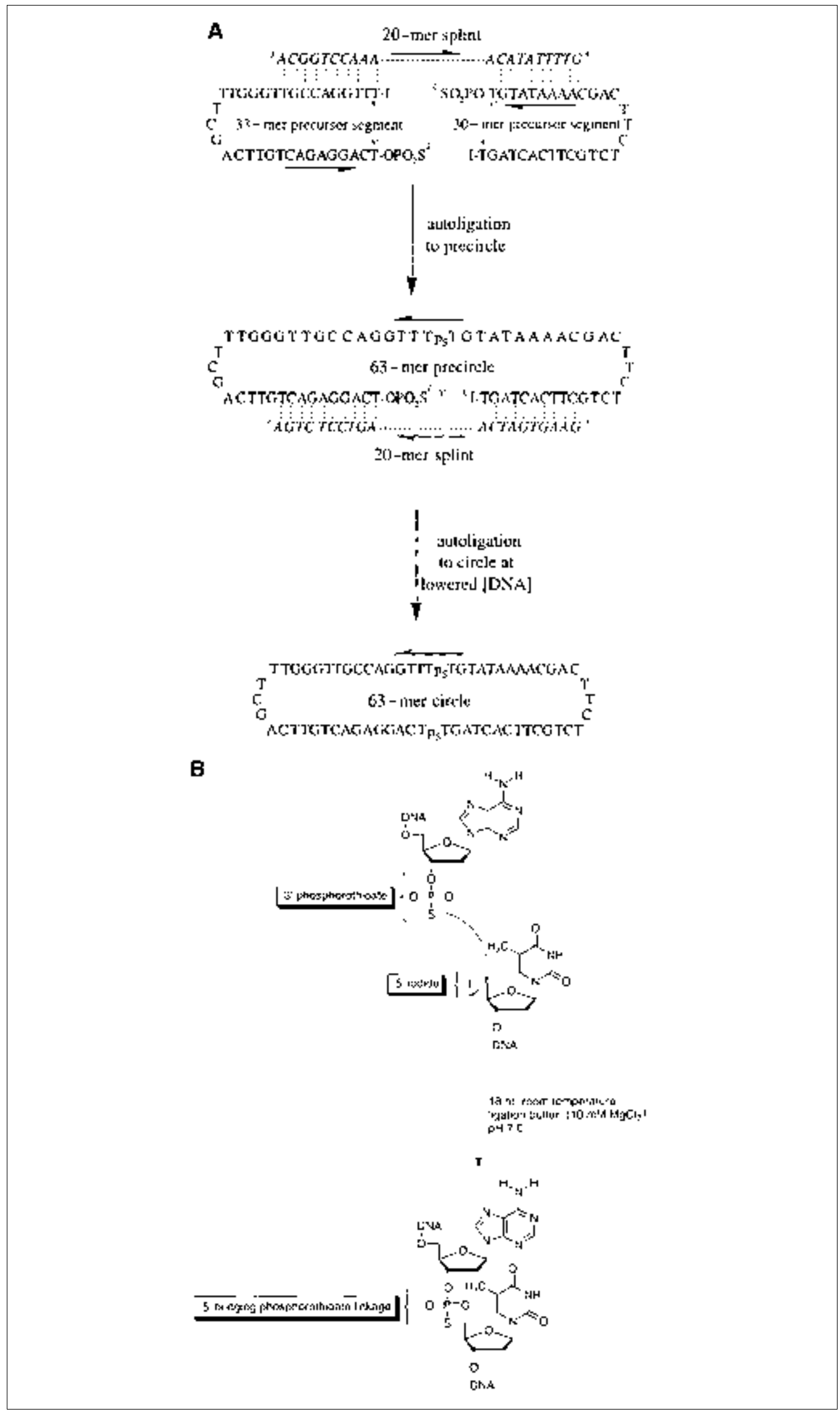

Chemical and Enzymatic Methods
Figure 5.2.7 Schematic representation of "one-pot" autoligation steps for the reagent-free cyclization of ssDNAs. (A) Complementary splints are used to form double-helical splint complexes to juxtapose reactive $3^{\prime}$-phosphorothioate and 5'-iodothymidine ends of linear ssDNAs for autoligation via nucleophilic attack by phosphorothioate on iodothymidine. (B) Basic chemistry of autoligation. 
length linear precursor segments, which are ligated to form a linear precircle and subsequently cyclized into a ssDNA circle (see Fig. 5.2.7). Circles $<50$ nt may be synthesized from a single precursor segment by a single ligation and circles $>140 \mathrm{nt}$ may be synthesized by ligation of more than two precursor segments; see Strategic Planning for more information. The products are separated by preparative denaturing PAGE and circularity confirmed by endonuclease cleavage.

NOTE: To synthesize larger quantities, the first ligation reaction can be scaled up. The second ligation reaction can also be scaled up, but multiple reaction tubes should be used rather than a larger-scale one-pot synthesis.

NOTE: If a single precursor segment is to be used (i.e., synthesis of a ssDNA circle $<50$ nt), skip steps 3 to 5 (i.e., omit the first ligation and proceed directly from quantitation to the second ligation described).

\section{Additional Materials (also see Basic Protocol)}

20 nt splint ssDNAs

Precursor segments containing a 3'-phosphorothioate and 5'-iodothymidine

$2 \times$ ligation buffer: $20 \mathrm{mM} \mathrm{MgCl} / 20 \mathrm{mM}$ Tris-acetate, $\mathrm{pH} 7.00$

\section{Obtain the ssDNAs}

1. Construct or purchase ssDNAs of the desired splint and precursor-segment sequences. Precursor segment oligos must be designed such that the chosen ligation junction contains a thymine on the $5^{\prime}$ side; this allows incorporation of the modified base, $5^{\prime}$-iodothymidine, for autoligation. Splint DNAs should bridge, by $10 \mathrm{nt}$ on each side, the gap between precursor segments for ligation (see Fig. 5.2.7A).

For ssDNAs purchased commercially, be sure splints are fully deprotected and precursor segments are fully deprotected and synthesized with a 5'-iodothymidine and $3^{\prime}$-phosphorothioate group. Alternatively, ssDNAs may be synthesized on a DNA synthesizer using the standard 0.2- $\mu \mathrm{mol}$ DNA cycle for splints and a slightly modified protocol for precursor segments and precircles; see Support Protocol 3 for 3'-phosphorothioate- and 5'-iodothymidine-containing precursor segments, and see Support Protocol 4 for deprotection.

\section{Quantitate DNA}

2. Quantitate DNA (see Basic Protocol, step 2).

\section{Perform first ligation}

3. Combine $125.0 \mu \mathrm{L}$ of $2 \times$ ligation buffer with $15 \mathrm{nmol}$ of each precursor segment and $18.3 \mathrm{nmol}$ of one of the splints (splint 1 ) in an autoclaved $1.5-\mathrm{mL}$ microcentrifuge tube.

IMPORTANT NOTE: For large DNA circles, $>140 \mathrm{nt}$, use all precursor segments and all but one splint in step 3.

Final concentrations: $10 \mathrm{mM} \mathrm{MgCl}, 10 \mathrm{mM}$ Tris.acetate, $60 \mu \mathrm{M}$ each precursor segment, $73 \mu$ M splint 1 .

It may not matter which splint is chosen for the first ligation here; however, more is required for the second ligation, so if one is limiting, it should be used here. If combined volume of DNA (two precursor segments and splint) exceeds $125.0 \mu \mathrm{L}$, one or more DNAs must be lyophilized to reduce the volume.

4. Calculate the volume of water to add according to the following equation:

volume water $=250 \mu \mathrm{L}-(125.0 \mu \mathrm{L}+$ volume DNA [step 3] $)$

Add the calculated amount of water to the $1.5-\mathrm{mL}$ tube, vortex briefly, and secure closed with a cap lock.

Methods for Cross-Linking Nucleic Acids 
The $250 \mu \mathrm{L}$ represents the total reaction volume, $125.0 \mu \mathrm{L}$ represents the $2 \times$ ligation buffer, and volume DNA represents the combined volumes of either precursor segments or precircle, plus the splint.

5. Incubate the capped tubes $18 \mathrm{hr}$ at room temperature.

\section{Perform second ligation}

6. In an autoclaved $15-\mathrm{mL}$ centrifuge tube, combine $5 \mathrm{~mL}$ of $2 \times$ ligation buffer with either $167.0 \mu \mathrm{L}(2 / 3$ volume $)$ of the reaction from step 5 or $10 \mathrm{nmol}$ full-length synthesized precursor segment if cyclization is from a single precursor segment, and $30 \mathrm{nmol}$ of remaining splint (splint 2).

Final concentrations: $10 \mathrm{mM} \mathrm{MgCl}, 10 \mathrm{mM}$ Tris.acetate, $1 \mu \mathrm{M}$ precircle, $3 \mu \mathrm{M}$ splint 2, $1.2 \mu$ M splint 1 .

IMPORTANT NOTE: For circles $>140$ nt, the final splint is added in this step.

The $167.0 \mu \mathrm{L}$ of reaction from step 5 represents, in theory, a maximum yield of $10 \mathrm{nmol}$ of precircle from ligated precursor segments. Alternatively, $10 \mathrm{nmol}$ of synthesized full-length precursor segment can be used if circle size is $<50$ nt. Note that DNA concentrations are much lower for this second ligation (1 $\mu \mathrm{M}$ precircle) than for the first $(60 \mu \mathrm{M}$ each precursor segment). This is meant to favor the intramolecular cyclization reaction versus an intermolecular reaction that produces multimeric linear species.

7. Calculate the volume of water to add according to the following equation:

volume water $=10,000 \mu \mathrm{L}-(5000 \mu \mathrm{L}+$ volume DNA [step 6] $)$

Add this volume of water to the 15 -mL tube(s), vortex briefly, and secure closed.

The 10,000 $\mu \mathrm{L}$ represents the total reaction volume, $5000 \mu \mathrm{L}$ represents the $2 \times$ ligation buffer, and volume DNA represents the combined volumes of either $167 \mu \mathrm{L}$ step 5 reaction or synthesized precircle, plus the splint. Normally it will not be necessary to be lyophilized any of the components at this step, as the calculated volume of water often exceeds $4.5 \mathrm{~mL}$.

8. Incubate the tubes $18 \mathrm{hr}$ at room temperature.

\section{Dialyze reaction}

9. Dialyze the reaction mixture from the second ligation (see Basic Protocol, steps 12 and 13).

\section{Gel purify DNA products}

10. Gel purify the dialyzed mixture (see Basic Protocol, steps 14 to 19).

The reaction lanes should show several bands. Figure 5.2.4 is a representation of a typical gel, with lane 6 containing cyclization mixture. Depending on how far the gel is run, the splints will probably be run off, but precursor segments may be visible and their identity can be confirmed by their co-migration with the splints and precursor-segment markers (step 2) run alongside (lanes 1 to 3). The next band up from precursor segments should be the precircle band and can be confirmed by co-migration with the largest band from the ligation reaction (step 5) run alongside (lane 4). There is the potential for non-unit-length linear multimers from the linear ligation of an odd number of precursor segments leading to products in-between precircle and circle (lane 7). Although DNA circles usually migrate more slowly than their linear counterparts (Serwer and Allen, 1984), identification of circles is not trivial. Circle migration is not always consistent and may be temperature dependent. Therefore, most bands migrating more slowly than confirmed precircle should be cut out for analysis. 
Isolate purified DNAs

11. Isolate purified DNAs from the electrophoretic gel (see Basic Protocol, steps 20 to 24).

Precircle and higher products should be isolated for reasons stated in step 10.

\section{Confirm circularity}

12. Confirm circularity of the desired DNA product (see Basic Protocol, steps 24 to 30).

\section{AUTOMATED SYNTHESIS OF PRECURSOR SEGMENTS CONTAINING 5'-PHOSPHATE FOR LIGATION}

The support protocol describes incorporation of 5'-phosphate end groups on precursor segments necessary for their use in the enzymatic ligation methodology presented in the Basic Protocol. While DNAs can be purchased containing the desired end groups, modification during automatic DNA synthesis is straightforward. Introduction of a $5^{\prime}$-phosphate is described.

NOTE: Precursor segments will contain a 3'-hydroxyl and 5'-phosphate after deprotection (see Support Protocol 4).

\section{Materials}

DNA synthesis reagents for $0.2-\mu \mathrm{mol}$ synthesis

Phosphorylating reagent (Chemical Phosphorylating Reagent, Glen Research)

DNA synthesizer with phosphoramidite chemistry

1. Synthesize all natural bases for precursor segments, with completion of the synthesis cycle retaining the $5^{\prime}$-DMT protecting group.

2. Place phosphorylating reagent in a synthesizer port.

3. Set up the synthesis cycle for normal DNA, using the DNA column from step 1 as the starting column. Enter the sequence with the first ( $\left.3^{\prime}\right)$ base as the last base added to the column in step 1 and the next added "nucleotide" as the port containing the phosphorylating reagent. Complete the synthesis cycle with DMT removal.

This adds a 5' phosphate to each of the precursor segments.

4. Proceed to deprotection (see Support Protocol 4).

\section{AUTOMATED SYNTHESIS OF PRECURSOR SEGMENTS CONTAINING 3'-PHOSPHATE FOR LIGATION}

The support protocol describes incorporation of 3 '-phosphate end groups on precursor segments necessary for their use in the chemical ligation methodology presented in Alternate Protocol 1. While DNAs can be purchased containing the desired end groups, modification during automatic DNA synthesis is straightforward. Introduction of a $3^{\prime}$-phosphate is described.

\section{Materials}

DNA synthesis reagents for $0.2-\mu \mathrm{mol}$ synthesis

Phosphorylating reagent (Chemical Phosphorylating Reagent, Glen Research)

DNA synthesizer with phosphoramidite chemistry

NOTE: This is analogous to addition of a $3^{\prime}$ phosphorothioate in Support Protocol 3, but standard oxidizing (rather than sulfurizing) conditions are used to afford a $5^{\prime}$-hydroxyl 
and 3'-phosphate, rather than a 3'-phosphorothioate, after deprotection (Support Protocol $4)$.

1. Begin synthesis of the precursor segment using any of the four base columns. Set up the synthesis cycle for normal DNA.

2. Place phosphorylating reagent in a synthesizer port.

3. Set up the synthesis cycle for normal DNA. Enter the sequence with the first ( $\left.3^{\prime}\right)$ base as the base located on the column and the next added "nucleotide" as the port containing phosphorylating reagent. Complete the cycle with DMT left on.

This adds a $5^{\prime}$-phosphate to the column.

Any normal base column can be used here, as deprotection of the finished product will cleave to the 5' side of this initially added phosphate group, thus cleaving the finished oligo from the 3' base present on the CPG bead. The DMT group should be left on for the next base to be added, which is the 3' base in the sequence.

4. Add the first $\left(3^{\prime}\right)$ nucleotide for the desired sequence to the column. Complete synthesis of the remaining bases on the oligo with removal of the DMT protecting group.

5. Proceed to deprotection (see Support Protocol 4).

SUPPORT PROTOCOL 3

Chemical and Enzymatic Methods

\section{AUTOMATED SYNTHESIS OF PRECURSOR SEGMENTS CONTAINING 3'-PHOSPHOROTHIOATE AND 5'-IODOTHYMIDINE FOR LIGATION}

This support protocol describes incorporation of 3 '-phosphorothioate and $5^{\prime}$-iodothymidine end groups on precursor segments necessary for their use in the autoligation chemistry presented in Alternate Protocol 2. While DNAs can be purchased containing the desired end groups, modification during automatic DNA synthesis is straightforward. Phosphorylation at the $3^{\prime}$ end, subsequent modification of this end group to a $3^{\prime}$-phosphorothioate, and introduction of a $5^{\prime}$-iodothymidine are described.

\section{Materials}

DNA synthesizer with phosphoramidite chemistry

DNA synthesis reagents for $0.2-\mu \mathrm{mol}$ synthesis

Phosphorylating reagent (S.1 in Fig. 5.2.8; Chemical Phosphorylating Reagent, Glen Research)

Sulfurizing reagent (S.3; Applied Biosystems)

Iodothymidine phosphoramidite (5'-I-dT-CE phosphoramidite, Glen Research)

NOTE: Precursor segments will contain a $5^{\prime}$-iodothymidine and a $3^{\prime}$-phosphorothioate rather than 5'-hydroxyl and 3'-hydroxyl after deprotection (see Support Protocol 4).

1. Begin synthesis of the precursor segment using any of the four normal base columns. Set up the synthesis cycle for normal DNA.

2. Place phosphorylating reagent in a synthesizer port.

3. Enter the sequence with the first ( $\left.3^{\prime}\right)$ base as the base located on the column and the next added "nucleotide" as the port containing phosphorylating reagent. Complete the cycle with DMT left on.

This adds a 5'-phosphate to the 3' base of the column.

Any base column can be used here, as deprotection of the finished product will cleave to the $5^{\prime}$ side of this initially added phosphate group (S.4), thus cleaving the finished oligo 


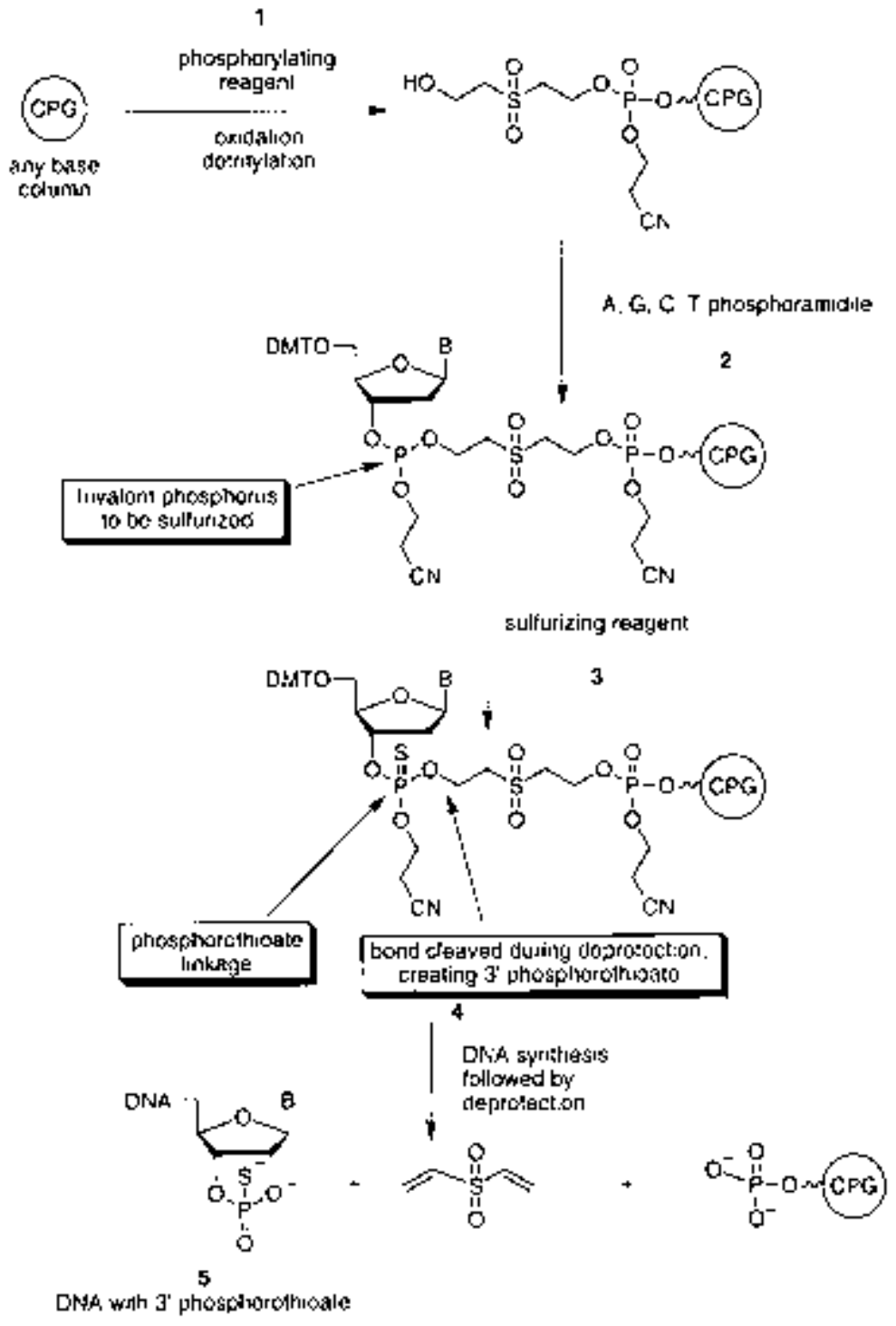

Figure 5.2.8 Schematic representation of steps involved in incorporation of a 3'-phosphorothioate group during DNA synthesis. First, a 3'-phosphate is coupled to the base on the CPG bead, S.1. Addition of the next base, S.2, the $3^{\prime}$ base of the desired sequence, proceeds with the trivalent phosphorus of this internucleotide bond converted to a phosphorothioate linkage using a sulfurizing reagent, S.3. Standard deprotection cleaves $3^{\prime}$ to this phosphorothioate linkage, S.4, creating a 3'-phosphorothioate group for coupling, S.5.

from the 3'-nucleotide present on the CPG bead (S.5). The DMT group should be left on for the next base to be added, which is the $3^{\prime}$ base in the sequence of interest. Alternatively, commercially available phosphate base columns can be used.

4. Set up the synthesizer to use a sulfurizing reagent (S.3) rather than an oxidizing reagent for this coupling only.

5. Proceed to add this next base and stop the synthesis after completion of this coupling.

This modified synthesis cycle (involving $\mathbf{S . 2}$ and $\mathbf{S . 3}$ ) adds the first (3') nucleotide for the sequence of interest to the column from step 3. The sulfurizing reagent converts the $3^{\prime}$ trivalent phosphorus linkage to a phosphorothioate linkage, S.4, rather than oxidized to a phosphodiester linkage as occurs during the standard DNA synthesis cycle. Subsequent

Methods for Cross-Linking Nucleic Acids 
deprotection will cleave $3^{\prime}$ to this linkage, leaving a 3'-phosphorothioate group as the nucleophile for autoligation (S.5).

6. Change the synthesis cycle back to standard (oxidizing agent instead of sulfurizing) and complete the synthesis of the desired precursor segments to the point at which synthesis of the natural bases (i.e., without iodothymidine) is complete, retaining the 5 -DMT protecting group.

IMPORTANT NOTE: Do not forget to change the synthesis cycle back to oxidizing from sulfurizing.

7. Place the modified phosphoramidite (5'-I-dT-CE phosphoramidite) in a synthesizer port.

8. Set up the synthesis cycle for normal DNA using the DNA column from step 5 as the starting column. Enter the sequence with the first ( $\left.3^{\prime}\right)$ base as the last base added to the column from step 5, and the next added "nucleotide" as the port containing the modified phosphoramidite. Complete the synthesis cycle without DMT removal.

This adds the modified phosphoramidite 5'-iodothymidine to each of the precursor segments. A DMT removal step is unnecessary because this phosphoramidite does not have a DMT protecting group.

9. Proceed to deprotection (see Support Protocol 4).

SUPPORT PROTOCOL 4

SUPPORT PROTOCOL 5

Chemical and Enzymatic Methods

\section{DEPROTECTION OF PRECURSOR SEGMENTS}

After completion of synthesis, the oligonucleotides must be cleaved from the solid support and protecting groups which are needed during synthesis must be removed. The procedure is different for precursor segments that do and do not contain $3^{\prime}$-phosphorothioate and 5 '-iodothymidine end groups in that gentler incubation conditions are used for the former.

\section{Materials}

Concentrated ammonium hydroxide

Pasteur pipets

Glass wool

1.5-mL screw-cap vial

1. Place DNA-loaded CPG beads from the column with $1 \mathrm{~mL}$ concentrated ammonium hydroxide in a $1.5-\mathrm{mL}$ screw-cap vial. Incubate $24 \mathrm{hr}$ at room temperature for precursors containing 3'-phosphorothioate and 5'-iodothymidine end groups, or 14 to $16 \mathrm{hr}$ at at $55^{\circ} \mathrm{C}$ for precursors not containing those groups.

2. Filter the solution through a glass wool-plugged pipet to separate CPG beads.

Use a second Pasteur pipet to transfer the solution.

3. Lyophilize the filtrate to remove ammonia and water. The crude DNA product should be powdery and white.

\section{PURIFICATION OF DEPROTECTED PRECURSOR SEGMENTS (OPTIONAL)}

Slightly increased product yields may be possible through the use of purified products. However, this will result in some product loss and is not needed, as each splint complexassisted ligation reaction mechanism selects for correct, full-length sequences, and the final product is gel purified to eliminate undesired DNAs. This support protocol is not recommended for precursor segments containing 3 -phosphorothioate or 5 '-iodothymid- 
ine end groups. The protocol describes a standard denaturing acrylamide gel method for purification of deprotected oligonucleotides.

CAUTION: Acrylamide and $N, N^{\prime}$-methylenebisacrylamide are neurotoxins. Preparation of solutions containing these compounds should be performed in a well-ventilated fume hood, and extreme precautions taken to minimize contact with solids or solutions.

\section{Materials}

$10 \%$ denaturing polyacrylamide gel mix (see recipe or purchase)

$10 \%(\mathrm{w} / \mathrm{v})$ ammonium persulfate (APS) in water

TEMED or TMEDA (Life Technologies)

$1 \times \operatorname{TBE}($ APPENDIX 2A)

Ultrapure (e.g., distilled, deionized) water

Formamide loading buffer (recipe)

$0.2 \mathrm{~N} \mathrm{NaCl}$

Dialysis tubing, MWCO 1000 (e.g., SpectraPor)

50-mL filter tubes with $0.45-\mu \mathrm{m}$ cellulose acetate filter (e.g., Spin-X II, Corning Costar)

Gel electrophoresis equipment:

Vertical (sequencing) gel stand

2000-V power supply

Glass plates: $13 \times 15.5 \mathrm{~cm}$ and $13 \times 16.5 \mathrm{~cm}$

2.5-mm-thick gel combs with 2.5 -cm-wide wells

2.5-mm-thick spacers

UV shadow box or light source

Lyophilizer or SpeedVac

Saran Wrap (or other UV-transparent plastic wrap)

Sterile razor blades

Glass stir rod

1. Prepare a vertical, 2.5 -mm-thick (preparative), denaturing $10 \%$ polyacrylamide gel using a comb producing 2.5 -cm-wide wells.

The well size is not critical, and the walls can even be removed to create one large well.

A gel prepared between plates used for sequencing $(13 \times 16.5 \mathrm{in}$.) allows for ample vertical loading of reaction mixture and sufficient migration length for separation and resolution of bands. For these conditions, prepare gel solution from $300 \mathrm{~mL}$ of $10 \%$ gel mix, $2 \mathrm{~mL}$ of $10 \%$ APS, and $64 \mu L T E M E D$. For more detailed description of procedures for denaturing PAGE, SEe APPENDIX $3 B$.

2. Place the gel in a gel electrophoresis apparatus, using $1 \times$ TBE in both top and bottom buffer reservoirs, and prerun $30 \mathrm{~min}$ at $30 \mathrm{~W}$ constant power, room temperature.

3. Redissolve each crude DNA (after deprotection and lyophilization) in $100 \mu \mathrm{L}$ water and $100 \mu \mathrm{L}$ formamide loading buffer by brief vortexing.

Synthesized DNA after deprotection and lyophilization should be powdery and white and readily redissolve in water. If the solution does not appear homogeneous, mild heating $\left(\sim 60^{\circ} \mathrm{C}\right)$ and/or brief sonication $(1 \mathrm{~min})$ can be used to help dissolve the DNA. Insoluble materials may cause some turbidity but are not cause for concern.

4. Load $25 \mu \mathrm{L}$ of solution from step 3 in each of the 2.5 -cm-wide wells; or, alternatively, remove well walls and load the entire sample in the large well created.

5. Run the gel at $30 \mathrm{~W}$ constant power, room temperature, to the desired length.

Methods for Cross-Linking Nucleic Acids

Current Protocols in Nucleic Acid Chemistry 
Bromphenol blue migrates similarly to a 26-nt linear DNA on a 10\% gel, and xylene cyanol runs like a 55-nt linear DNA.

6. Disassemble the gel apparatus, separate the plates, and sandwich the gel between two pieces of Saran Wrap. View the gel under ultraviolet light with fluorescent white background (glass silica plates work well) to visualize the DNA.

There should be an intense, slow-migrating band, which is the desired sequence, as well as faster-migrating shorter sequences below.

7. Cut out the desired band with a clean, sterile razor blade and place the cut up pieces in three or four $15-\mathrm{mL}$ centrifuge tubes. Using a glass stir rod, crush the gel pieces thoroughly. Combine crushed gel pieces in each tube with $\sim 5$ to $10 \mathrm{~mL}$ of $0.2 \mathrm{~N} \mathrm{NaCl}$ to make an easily shaken slurry. Repeat, in separate tubes, for each band isolated. Shake each slurry for $\sim 12 \mathrm{hr}$.

Note that high-purity DNAs can be obtained by individually crushing thin vertical portions of the large piece isolated above. Be sure all crushed gel pieces are transferred to the slurry, as isolated yields are often $<50 \%$, and it is therefore important to ensure a quantitative transfer of all materials.

8. Filter the slurry by brief centrifuging 1 to $5 \mathrm{~min}$ through $50-\mathrm{mL}$ filter tubes. Resuspend the gel pieces in $5 \mathrm{~mL}$ water, centrifuge again, and combine the filtrates.

It is advisable to save the filtered gel pieces until after quantitation, as additional extractions with $0.2 \mathrm{~N} \mathrm{NaCl}$ may be desired to increase isolated product yield, although only minimal $(<10 \%)$ further recoveries should be expected.

9. Transfer the entire reaction volume from step 8 to MWCO 1000 dialysis tubing clamped at one end to make a bag. Secure the other end of the dialysis bag closed. Dialyze against $2 \mathrm{~L}$ of ultrapure water for $3 \mathrm{hr}$. Repeat.

10. Lyophilize the dialysate to dryness.

The dried residue should powdery and white.

\section{REAGENTS AND SOLUTIONS}

Use distilled, deionized water or other ultrapure water in all recipes and protocol steps. For common stock solutions, see APPENDIX 2A; for suppliers, see SUPPLIERS APPENDIX. Most of these reagents can be purchased premade from a biological supply company.

\section{Denaturing polyacrylamide gel mix, $10 \%$}

Dissolve $450 \mathrm{~g}$ urea, $95.0 \mathrm{~g}$ acrylamide, and $5.00 \mathrm{~g} N, N^{\prime}$-methylenebisacrylamide in $100.0 \mathrm{~mL}$ of $10 \times \mathrm{TBE}$ (APPENDIX $2 A$ ) diluted to a final volume of $1.00 \mathrm{~L}$ with water. Filter using $0.45-\mu \mathrm{m}$ nylon filter paper.

CAUTION: Acrylamide and N,N'-methylenebisacrylamide are neurotoxins. Preparation of solutions with these compounds should be performed in a well-ventilated fume hood, and extreme precautions taken to minimize contact with compounds or solutions.

\section{Formamide loading buffer}

Dissolve $10.0 \mathrm{~mL}$ of $10 \mathrm{mM}$ EDTA, pH 8.0 (APPENDIX $2 A$ ), in $40 \mathrm{~mL}$ formamide. Add $5.00 \mathrm{mg}$ each bromophenol blue and xylene cyanol dyes.

Chemical and Enzymatic Methods

\section{Stains-all dye solution}

Dissolve $20.0 \mathrm{mg}$ stains-all (3,3'-diethyl-9-methyl-4,5,4',5'-debenzothiacarbocyanine; Sigma) in $25.0 \mathrm{~mL}$ formamide and dilute to $200 \mathrm{~mL}$ with water. 
Dissolve $5.77 \mathrm{~g}$ urea, $1.2 \mathrm{mg}$ each bromophenol blue and xylene cyanol, and 720 $\mu \mathrm{L}$ of a stock (500 mM) EDTA solution in water to a final volume of $12 \mathrm{~mL}$.

Final concentrations are $8 \mathrm{M}$ urea, $30 \mathrm{mM}$ EDTA, and $0.1 \mathrm{mg} / \mathrm{mL}$ of each gel running dye.

\section{COMMENTARY}

\section{Background Information}

Circularized single-stranded deoxyribonucleotides (ssDNAs) are more resistant to nuclease activity than their linear counterparts (Rumney and Kool, 1992), a property that may be important for in vivo studies. Circular DNAs have been investigated for their unique DNA binding properties (Wang and Kool, 1994; Kool, 1996) and also as useful models in studying DNA structures such as hairpin motifs by NMR (Ippel et al., 1995). DNA circles are also currently being used for more diagnostic applications, such as the creation of padlock probes (Nilsson et al., 1994, 1997). Circular oligonucleotide templates have also been used for the synthesis of concatemeric polypeptides (Brown, 1997). Additionally, circular ssDNAs offer a novel means of both DNA and RNA amplification as these molecules are accepted as templates by both DNA and RNA polymerases (Fire and Xu, 1995; Liu et al., 1996; Daubendiek and Kool, 1997).

Circular ssDNAs have been constructed using a range of chemical, enzymatic, and reagent-free ligation methods. Chemical ligation using cyanogen bromide has been effective for the synthesis of circles from triple-helical (Ruben et al., 1995) as well as double-helical splint complexes (Dolinnaya et al., 1993). Chemical ligation using carbodiimide chemistry (Ashley and Kushlan, 1991) is becoming a less common method of ligation despite reported higher yields as compared to cyanogen bromide (Dolinnaya et al., 1991), possibly because of the long reaction times required. Enzymatic ligation, which gives comparable yields albeit with higher cost, eliminates the need for either of these chemicals, which may be damaging to DNA. Novel approaches to the synthesis of small circular DNAs are becoming more common as solid-phase methodology improves (De Napoli et al., 1995; Alazzouzi et al., 1997). Of great interest are new autoligation, or reagent-free, methods (Herrlein and Letsinger, 1994; Herrlein et al., 1995), since these eliminate the need for a separate ligation reagent. The finite stability of the tosyl-modified DNAs required for these couplings limits the methods' practical applications. A recent report by Xu and Kool (1997) describes a novel 5'-iodonucleoside that eliminates this stability issue. While this method produces a non-natural bridging phosphorothioate, the transcription and replication ability of the DNAs does not appear to be affected, as the circles were transcribed and replicated successfully in a rollingcircle fashion (Y. Xu and E.T. Kool, unpub.observ.). It is noteworthy that this bridging $5^{\prime}$ phosphorothioate linkage in DNA is stable for at least several months in the $\mathrm{pH}$ range 5 to 9 .

Each of the three protocols in this unit is potentially applicable to the synthesis of circles ranging in size from $28 \mathrm{nt}$ to at least $188 \mathrm{nt}$; however, sequence and size may render one or another method more suitable in specific cases. The enzymatic ligation method (see Basic Protocol) has few sequence or size restrictions, though small circles, and larger circles that may have sequence-induced secondary structure, may not be good substrates for the enzyme. The chemical ligation approach using cyanogen bromide (see Alternate Protocol 1) has been very effective in the creation of pyrimidine-rich circles by ligation of triple-helical splint complexes (Ruben et al., 1995). It has not, however, proven effective to the authors' satisfaction in creating DNA circles from a double-helical splint complex using the methodology described by Dolinnaya et al. (1993). The phosphorothioate autoligation method (see Alternate Protocol 2) requires segment precursors to contain a $5^{\prime}$-thymidine (from iodothymidine) and a $3^{\prime}$-phosphorothioate group at the ligation junction.

For larger circles, another issue arises: the tradeoff between optimized ligation yields and DNA synthesis yields. Longer synthesized DNAs will be produced in lower yields than shorter ones, but ligation of shorter oligos may proceed in higher yields than their longer counterparts. Consider the synthesis of a 100-mer ssDNA circle. While an average stepwise coupling yield of $98 \%$ on the synthesizer results in $37 \%$ overall yield of each 50-mer precursor segment, the same coupling yields give only $14 \%$ of the full-length 100 -mer precircle. If the single ligation of precircle to circle proceeds in a $40 \%$ overall yield, and the double ligation
Methods for Cross-Linking Nucleic Acids 
only in 30\% overall yield, the overall yield of circle from this "convergent" (double-ligation) approach is still 2-fold greater. So in planning strategically for cyclization, one should take into account circle size and optimization of both DNA synthesis and ligation when designing starting materials.

Enzymatic ligation proceeds via a $3^{\prime}$-hydroxyl and 5'-phosphate, affording a "natural" phosphodiester backbone. However, this "natural" method comes with an expensive price-tag and, with larger circles (>100 nt), potentially low yields. While the cyanogen bromide method uses the same starting materials for ligation, also yielding the desired "natural" linkage, the reagents for ligation, while substantially cheaper, may be moderately caustic to DNA. So although yields may be comparable to those from enzymatic ligation, the benefit of cheaper reagents may not outweigh the cost of possible product damage. The autoligation method has a cost advantage, since it eliminates the need for a separate ligation reagent, but creates a non-natural phosphorothioate linkage.

\section{Compound Characterization}

As described in each individual protocol, confirmation of product circularity is quite rapid and simple and may be of critical importance when considering future applications. The analysis of this characterization is quite unambiguous, as described in the sections within each protocol on confirmation of circularity. Initial cleavage of a circle produces a single band with the mobility of the full-length cyclization precursor (Fig. 5.2.5). If the putative circular DNA is actually linear, banding will be seen directly under the suspected band. This type of banding should also be seen for the precircle reaction lane. This reaction, and/or one analogous to it, must be completed to ensure product quality.

\section{Critical Parameters}

There are few complicating factors with these reactions, although several potential problems will be addressed in the troubleshooting section. However, there are a few "critical parameters" worth mentioning. For all syntheses, dialysis of the final ligation-reaction mixture to remove all salts prior to lyophilization and gel purification is critical, as excess salts can cause problems with gel migration. It is worthwhile to note that for all reactions done via a double ligation (i.e., with two precursor segments), the concentration of precircle in the second step is purposely lowered to favor this intramolecular ligation producing circles as compared to the intermolecular ligation creating linear multimers.

Another point to note is that while both enzymatic and autoligation occur via the same double-helical splint complex, only the enzymatic ligation protocol calls for slow annealing of precursor DNAs and splints prior to addition of ligation reagents (steps 6 and 10 of the Basic Protocol). This simply represents different methodologies consistently used in the authors' lab, and while enzymatic ligation may proceed just as efficiently without these steps, this asyet-unexplored option has not been described in the protocol. For enzymatic ligation, the enzyme should be stored at $-20^{\circ} \mathrm{C}$ prior to its use, and reaction temperature should be maintained consistently at $\leq 37^{\circ} \mathrm{C}$, as higher temperatures may cause enzyme denaturation and inactivation.

The only special precaution when using cyanogen bromide ligation is that, since this chemical is quite toxic and volatile, appropriate care must be taken when using it. By much the same token, the reaction should be stopped promptly when instructed, as prolonged exposure may increase the DNA's susceptibility to damage.

The only specific considerations for the phosphorothioate method are in the synthesis of the modified precursor segments. It is important to follow the directions for synthesis exactly, first phosphorylating the initial base column with subsequent addition of the desired $3^{\prime}$ base and sulfurization of only this internucleotide linkage. As for the 5 -iodothymidine phosphoramidite, this particular compound is unique-compared to other similar compounds designed to ligate ssDNAs via an $\mathrm{S}_{\mathrm{N}} 2$ mechanism-in having greatly enhanced stability, which significantly extends the shelf lives of both the phosphoramidite and the modified precursor-segment DNAs (Xu and Kool, 1997).

For a discussion of common problems arising with these procedures, including their diagnosis and possible solutions, see Table 5.2.1.

\section{Anticipated Results}

For all three methods, optimized yields after purification are modest, and often less than the observed qualitative conversion. For circles of $\sim 65 \mathrm{nt}$, yields of $33 \%$ for enzymatic ligation (Diegelman, pers. comm.) and 20\% for autoligation (Xu et al., 1997) have been reported along with a comparable yield of $30 \%$ for 
Table 5.2.1 Troubleshooting Guide for Synthesis of Circular DNAs

\begin{tabular}{|c|c|c|}
\hline Problem & Possible cause & Possible solution \\
\hline \multirow[t]{3}{*}{ Poor DNA synthesis yields } & Reagents contaminated with water & $\begin{array}{l}\text { Ensure reagents are anhydrous (distill } \\
\text { solvents fresh) }\end{array}$ \\
\hline & Glass frits in synthesizer dirty & $\begin{array}{l}\text { Boil } 15 \mathrm{~min} \text { in water and sonicate } 30 \mathrm{~min} \text { in } \\
\text { methanol }\end{array}$ \\
\hline & Phosphoramidites degraded & Use new phosphoramidites \\
\hline \multirow[t]{2}{*}{ Poor phosphorylation yields } & Reagents contaminated with water & $\begin{array}{l}\text { Distill fresh } \mathrm{CH}_{3} \mathrm{CN} \text { to prepare } \\
\text { phosphorylating reagent }\end{array}$ \\
\hline & Phosphoramidites degraded & Purchase fresh reagents \\
\hline $\begin{array}{l}\text { Crude DNA not dissolving } \\
\text { in water }\end{array}$ & $\begin{array}{l}\text { Insoluble material found after } \\
\text { deprotection }\end{array}$ & $\begin{array}{l}\text { Briefly heat at } 60^{\circ} \mathrm{C} \text { and sonicate to dissolve } \\
\text { DNA or briefly centrifuge crude DNA and } \\
\text { work with supernatant }\end{array}$ \\
\hline \multirow[t]{6}{*}{$\begin{array}{l}\text { No/low absorbance seen for } \\
\text { DNA quantitation }\end{array}$} & Measurement not at correct wavelength & $\begin{array}{l}\text { Set absorbance at } 260 \mathrm{~nm} \text {; check that UV } \\
\text { lamp is working properly }\end{array}$ \\
\hline & Cuvette dirty or not inserted properly & $\begin{array}{l}\text { Ensure cuvette is clean and unscratched and } \\
\text { light passes through its clear sides }\end{array}$ \\
\hline & Instrument not properly zeroed & $\begin{array}{l}\text { Ensure instrument is properly zeroed with a } \\
\text { cuvette containing only water }\end{array}$ \\
\hline & Instrument not properly aligned & $\begin{array}{l}\text { Consult spectrophotometer manual on } \\
\text { ensuring unimpeded light path }\end{array}$ \\
\hline & No DNA in sample & $\begin{array}{l}\text { See "Crude DNA not dissolving in water," } \\
\text { above }\end{array}$ \\
\hline & $\begin{array}{l}\text { DNA concentration low after isolation } \\
\text { of products from gel pieces }\end{array}$ & $\begin{array}{l}\text { If quantitating gel-purified products, try } \\
\text { re-extracting the slurry mixture with } 0.2 \mathrm{~N} \\
\mathrm{NaCl}\end{array}$ \\
\hline \multirow[t]{7}{*}{$\begin{array}{l}\text { No ligation seen by UV } \\
\text { shadowing }\end{array}$} & $\begin{array}{l}\text { One or more DNAs (especially splint) } \\
\text { omitted from reaction mixture }\end{array}$ & $\begin{array}{l}\text { Rerun reaction, ensuring addition of all } \\
\text { DNAs needed }\end{array}$ \\
\hline & $\begin{array}{l}\text { Enzyme old or inactive (enzymatic } \\
\text { ligation) }\end{array}$ & $\begin{array}{l}\text { Rerun reaction using a fresh batch of } \\
\text { enzyme, and ensure proper storage }\left(-20^{\circ} \mathrm{C}\right) \\
\text { after use }\end{array}$ \\
\hline & $\begin{array}{l}\text { DTT or BrCN degraded (enzymatic } \\
\text { ligation) }\end{array}$ & $\begin{array}{l}\text { Rerun reaction using fresh DTT or } \mathrm{BrCN} \\
\text { stock }\end{array}$ \\
\hline & $\begin{array}{l}\text { Reaction allowed to proceed too long } \\
\text { (chemical ligation) }\end{array}$ & Rerun reaction for shorter period \\
\hline & $\begin{array}{l}\text { DNA modification (phosphorylation) } \\
\text { poor, leading to reduced ligation yield } \\
\text { (enzymatic and chemical ligation) }\end{array}$ & See "Poor phosphorylation yields," above \\
\hline & $\begin{array}{l}\text { Poor incorporation of the modified base, } \\
5^{\prime} \text {-iodothymidine (autoligation) }\end{array}$ & $\begin{array}{l}\text { Ensure coupling of modified } \\
\text { phosphoramidite and sulfurization were } \\
\text { effective }^{b} \text {; rerun reaction, ensuring DNA } \\
\text { synthesizer cycle is set up properly }\end{array}$ \\
\hline & Poor splint hybridization & $\begin{array}{l}\text { Rerun reaction; possibly redesign new splint } \\
\text { junction (sequence context problem may } \\
\text { exist) }\end{array}$ \\
\hline \multirow{2}{*}{$\begin{array}{l}\text { Isolated product not } \\
\text { behaving as expected for } \\
\text { circular DNA }\end{array}$} & Isolated product is not a circular DNA & $\begin{array}{l}\text { Rerun S1 nuclease reaction on other isolated } \\
\text { bands }\end{array}$ \\
\hline & $\begin{array}{l}\text { Isolated product is circular but has } \\
\text { degraded significantly (less likely; check } \\
\text { for circularity first) })^{a}\end{array}$ & $\begin{array}{l}\text { Resynthesize DNA, taking greater } \\
\text { precautions to avoid degradation }\end{array}$ \\
\hline
\end{tabular}

${ }^{a}$ Common forms of damage are formation of abasic sites from depurination during synthesis, and degradation by reagents (e.g., $\left.\mathrm{BrCN}\right)$ or heat or acid treatments.

${ }^{b}$ Sulfurization can be examined by electrospray or MALDI-TOF MS (UNITS $10.1 \& 10.2$ ). Iodothymidine incorporation can be checked by comparing the oligonucleotide to one without iodo-T added using either high-resolution sequencing PAGE (APPENDIX 3B) or C18-reversed-phase HPLC. 
cyanogen bromide ligation of a 42-nt triplexforming circle (Ruben et al., 1995). For all syntheses involving more than one precursor segment and splint, and methodology involving a double ligation (two step, one pot) rather than a dimerization approach (triple-helical splintcomplex ligation with cyanogen bromide), intermediate, non-unit-length products (besides the desired circles) are often seen.

In general, the observed yield-as determined by UV shadowing to estimate product conversion-is often twice the isolated yield. This apparently represents the inability to completely extract product from gel slurry; reextraction with additional eluent $(0.2 \mathrm{~N} \mathrm{NaCl})$ may increase product yields somewhat, although only minimal $(<10 \%)$ recoveries should be expected.

\section{Time Considerations}

Completion of any of the above three protocols should take no longer than 7 days, and most can be completed in less time either by purchasing synthesized DNAs or by using fast-deprotection phosphoramidites. Actual hands-on work occupies much less total time, but several waiting periods are required. Synthesis of oligos, with overnight deprotection, followed by lyophilization and quantitation the next day should take $\sim 1.5$ days. A double ligation set up to run the remainder of day 2 with the second ligation running overnight should take another 1.5 days, resulting in completion of the ligations by the middle of day 3. Dialysis for $6 \mathrm{hr}$ and lyophilization overnight allows gel purification on day 4 and elution from gel pieces overnight to the morning of day 5. Following a 24-hr dialysis, the circular products should be ready partway through day 6 for quantitation and structural confirmation experiments.

\section{Literature Cited}

Alazzouzi, E., Escaja, N., Grandas, A., and Pedroso, E. 1997. A straightforward solid-phase synthesis of cyclic oligodeoxyribonucleotides. Angew. Chem. Int. Ed. Engl. 36:1506-1508.

Ashley, G.W. and Kushlan, D.M. 1991. Chemical synthesis of oligodeoxynucleotide dumbbells. Biochemistry 30:2927-2933.

Borer, P.N. 1975. Optical properties of nucleic acids. In Handbook of Biochemistry and Molecular Biology, Vol. I, 3rd ed. (G.D. Fasman, ed.) p. 589. CRC Press, Boca Raton, Fla.

Brown, S. 1997. Metal-recognition by repeating polypeptides. Nature Biotechnol. 15:269-272.
Daubendiek, S.L. and Kool, E.T. 1997. Generation of catalytic RNAs by rolling transcription of synthetic DNA nanocircles. Nature Biotechnol. 15:273-277.

De Napoli, L., Galeone, A., Mayol, L., Messere, A., Montesarchio, D., and Piccialli, G. 1995. Automatic solid phase synthesis of cyclic oligonucleotides: A further improvement. Bioorg. Med. Chem. 3:1325-1329.

Dolinnaya, N.G., Sokolova, N.I., Ashirbekova, D.T., and Shabarova, Z.A. 1991. The use of BrCN for assembling modified DNA duplexes and DNARNA hybrids: Comparison with water-soluble carbodiimide. Nucl. Acids Res. 9:3067-3072.

Dolinnaya, N.G., Blumenfeld, M., Merenkova, I.N., Oretskaya, T.S., Krynetskaya, N.F., Ivanovskaya, M.G., Vasseur, M., and Shabarova, Z.A. 1993. Oligonucleotide circularization by splint-directed chemical ligation. Nucl. Acids Res. 21:5403-5407.

Fire, A. and Xu, S.Q. 1995. Rolling replication of short DNA circles. Proc. Natl. Acad. Sci. U.S.A. 92:4641-4645.

Herrlein, M.K. and Letsinger, R.L. 1994. Selective chemical autoligation on a double-stranded DNA splint. Nucl. Acids Res. 22:5076-5078.

Herrlein, M.K., Nelson, J.S., and Letsinger, R.L. 1995. A covalent lock for self-assembled oligonucleotide conjugates. J. Am. Chem. Soc. 117:10151-10152.

Ippel, J.H., Lanzotti, V., Galeone, A., Mayol, L., Van den Boogaart J.E., Pikkemaat, J.A., and Altona, C. 1995. Slow conformational exchange in DNA minihairpin loops: A conformational study of the circular dumbbell d $<$ pCGC-TT-GCG-TT $>$. Biopolymers 36:681-694.

Kool, E.T. 1996. Circular oligonucleotides: New concepts in oligonucleotide design. Annu. Rev. Biophys. Biomol. Struct. 25:1-28.

Liu, D., Daubendiek, S.L., Zillman, M.A., Ryan, K., and Kool, E.T. 1996. Rolling circle DNA synthesis: Small circular oligonucleotides as efficient templates for DNA polymerases. J. Am. Chem. Soc. 118:1587-1594.

Nilsson, M., Malmgren, H., Samiotaki, M., Kwiatkowski, M., Chowdhary, B.P., and Landegren, U. 1994. Padlock probes: Circularizing oligonucleotides for localized DNA detection. Science 265:2085-2088.

Nilsson, M., Krejci, K., Koch, J., Kwiatkowski, M., Gustavsson, P., and Landegren, U. 1997. Padlock probes reveal single-nucleotide differences, parent of origin and in situ distribution of centromeric sequences in human chromosomes 13 and 21. Nature Genet. 16:252255.

Ruben, E., Rumney, S. IV, Wang, S., and Kool, E.T. 1995. Convergent DNA synthesis: A non-enzymatic dimerization approach to circular oligodeoxynucleotides. Nucl. Acids Res. 23:3547-3553. 
Rumney, S. IV and Kool, E.T. 1992. DNA recognition by hybrid oligoether-oligodeoxynucleotide macrocycles. Angew. Chem. Int. Ed. Engl. 31:1617-1619.

Serwer, P. and Allen, J.L. 1984. Conformation of double-stranded DNA during agarose gel electrophoresis: Fractionation of linear and circular molecules with molecular weights between $3 \times$ $10^{6}$ and $26 \times 10^{6}$. Biochemistry 23:922-927.

Wang, S. and Kool, E.T. 1994. Circular RNA oligonucleotides. Synthesis, nucleic acid binding properties, and a comparison with circular DNAs. Nucl. Acids Res. 22:2326-2333.

$\mathrm{Xu}$, Y. and Kool, E.T. 1997. A novel 5'-iodonucleoside allows efficient nonenzymatic ligation of single-stranded and duplex DNAs. Tetrahedron Lett. 38:5595-5598.

\section{Key References}

Alazzouzi et al., 1997. See above.

Reports a unique methodology for the solid-phase synthesis of small $(<32 \mathrm{nt})$ ssDNA circles. No splint is needed, but yields are low for the larger circles in this size range.
Dolinnaya et al., 1993. See above.

Reports the synthesis of medium-sized ( 40 nt) ssDNA circles from a double-helical splint complex with cyanogen bromide. Certain sequence requirements for successful ligation are described.

Ruben et al., 1995. See above.

Reports the cyanogen bromide-mediated synthesis of medium-sized (34- to 72-nt) ssDNA circles from two short segments using a triple-helical splint complex.

Xu and Kool, 1997. See above.

Reports an autoligation method incorporating a novel 5'-iodothymidine phosphoramidite (now commercially available) which possesses increased stability as compared to one previously described (Herrlein et al., 1995).

Contributed by Amy M. Diegelman and Eric T. Kool

University of Rochester

Rochester, New York
Methods for Cross-Linking Nucleic Acids 